\title{
TNF and granulocyte macrophage-colony stimulating factor interdependence mediates inflammation via CCL17
}

\author{
Andrew D. Cook, ${ }^{1}$ Ming-Chin Lee, ${ }^{1}$ Reem Saleh, ${ }^{1}$ Hsu-Wei Khiew, ${ }^{1}$ Anne D. Christensen, ${ }^{1}$ \\ Adrian Achuthan, ${ }^{1}$ Andrew J. Fleetwood, ${ }^{1}$ Derek C. Lacey, ${ }^{1}$ Julia E. Smith, ${ }^{2}$ Irmgard Förster, ${ }^{3}$ \\ and John A. Hamilton ${ }^{1}$ \\ 'University of Melbourne, Department of Medicine, Royal Melbourne Hospital, Parkville, Victoria, Australia. ${ }^{2}$ Cytokine, \\ Chemokine and Complement DPU, Immunoinflammation TA, GSK Medicines Research Centre, Stevenage, Hertfordshire, \\ United Kingdom. ${ }^{3}$ mmunology and Environment, Life and Medical Sciences Institute University of Bonn, Bonn, Germany.
}

TNF and granulocyte macrophage-colony stimulating factor (GM-CSF) have proinflammatory activity and both contribute, for example, to rheumatoid arthritis pathogenesis. We previously identified a new $\mathrm{CM}-\mathrm{CSF} \rightarrow$ JMJD3 demethylase $\rightarrow$ interferon regulatory factor 4 (IRF4) $\rightarrow$ CCL17 pathway that is active in monocytes/macrophages in vitro and important for inflammatory pain, as well as for arthritic pain and disease. Here we provide evidence for a nexus between TNF and this pathway, and for TNF and CM-CSF interdependency. We report that the initiation of zymosaninduced inflammatory pain and zymosan-induced arthritic pain and disease are TNF dependent. Once arthritic pain and disease are established, blockade of CM-CSF or CCL17, but not of TNF, is still able to ameliorate them. TNF is required for CM-CSF-driven inflammatory pain and for initiation of GM-CSF-driven arthritic pain and disease, but not once they are established. TNFdriven inflammatory pain and TNF-driven arthritic pain and disease are dependent on CM-CSF and mechanistically require the same downstream pathway involving CM-CSF $\rightarrow$ CCL17 formation via JMJD3-regulated IRF4 production, indicating that CM-CSF and CCL17 can mediate some of the proinflammatory and algesic actions of TNF. Given we found that TNF appears important only early in arthritic pain and disease progression, targeting a downstream mediator, such as CCL17, which appears to act throughout the course of disease, could be effective at ameliorating chronic inflammatory conditions where TNF is implicated.

Conflict of interest: JES is an employee of CSK Research and Development Limited.

Submitted: December 13, 2017

Accepted: February 20, 2018

Published: March 22, 2018

Reference information:

JCI Insight. 2018;3(6):e99249.

https://doi.org/10.1172/jici.

insight.99249.

\section{Introduction}

Even though anti-TNF therapies have been known to be quite successful for some time in many patients with inflammatory disease, such as rheumatoid arthritis (RA), its mode of action is still unclear (1) - such information would be quite useful in developing improved therapies and predicting patient responses. Granulocyte macrophage-colony stimulating factor (GM-CSF) also has proinflammatory activity and there are promising signs that its blockade and that of its receptor are effective in RA trials, as well as in preclinical models (2-4). For example, in a phase IIb trial, treatment of RA patients with mavrilimumab, a neutralizing $\mathrm{mAb}$ targeting the GM-CSF receptor $\alpha$ chain, led to a rapid improvement in their assessment of pain within 1 week of treatment (5). Whether or not the same or different patient populations respond to the respective therapies targeting the action of TNF and GM-CSF is unknown and more needs to be learned about the extent of the overlap or not between their respective biologies with implications for therapeutic strategies. There are differences with respect to the expression and mode of action of the 2 cytokines $(1,6)$. Their receptors are not expressed on identical cell populations, with the TNF receptor expressed widely and the GM-CSF receptor usually expressed on myeloid populations; the signaling pathways downstream of their receptors also vary with, for example, NF-kB being important for TNF signaling and JAK/ STAT pathways for GM-CSF responses (1).

In spite of these differences, there is evidence that there are links between the 2 cytokines (7-9). They have also been used in partnership for the in vitro differentiation of dendritic cells from precursor cells (10). In vitro studies, mainly with monocyte(s)/macrophages, have shown that GM-CSF can upregulate TNF 
expression, at least at the mRNA level (11), while inversely TNF can induce GM-CSF formation and secretion in a number of tissue cell populations, such as fibroblasts, endothelial cells, and chondrocytes (12-15); in line with the latter type of studies, TNF neutralization of ex vivo RA synovial cell cultures led to reduced GM-CSF levels (7). These findings gave rise to the concepts that TNF and GM-CSF may, in fact, be operating within a cytokine hierarchy $(7,8)$ or a cytokine network, the latter concept perhaps helping to explain the chronicity of certain inflammatory lesions $(2,16-18)$.

Supporting the above-mentioned RA data, TNF and GM-CSF have both been found to be important in the progression of a number of preclinical inflammation/autoimmune models, including arthritis and its associated pain $(1-4,19)$. They have also been implicated in inflammatory pain models (20-22). Recently, we found that $\mathrm{Ab}$-mediated neutralization of GM-CSF receptor $\alpha$ chain and TNF depleted the inflammatory cell populations differently in arthritis and peritonitis models (6). Also, a new GM-CSF- and IRF4dependent pathway leading to CCL17 formation in inflammation was proposed that appears to be separate from the pathway(s) governing TNF formation in human and murine monocytes/macrophages (23).

Given the above literature, we explored further the relationship between TNF and GM-CSF in models of inflammatory pain and arthritic pain/disease. We report that in the models tested, TNF and GM-CSF are interdependent, both being required for the induction of inflammatory pain and arthritis development; however, once arthritic pain and disease are established, TNF is no longer required, unlike GM-CSF. Furthermore, TNF-driven inflammatory pain and a new TNF-driven arthritic pain and disease model require mechanistically the same downstream pathway as GM-CSF-driven pain and arthritis, involving GM-CSFdependent CCL17 formation via JMJD3-regulated IRF4 production (23).

\section{Results}

TNF is also required in models of GM-CSF- and CCL17-dependent inflammatory pain and in models of GM-CSFand CCL17-dependent arthritic pain and disease. We have shown inflammatory pain following intraplantar (i.pl.) zymosan injection, as well as arthritic pain and disease following intra-articular (i.a.) zymosan injection, to be dependent on GM-CSF, as well as on IRF4 and CCL17 (23). Tnfr $1^{-1-}$ mice are reported to show reduced pain compared with WT mice following i.a. zymosan injection (24).

Using $\mathrm{Tnf}^{\prime-}$ mice we firstly determined that i.pl. zymosan-induced pain, as measured by a change in weight distribution (using the well-validated incapacitance meter method (23, 25-27), was TNF dependent (Figure 1A), with some reduced footpad swelling seen at 5-6 hours after injection (Figure 1B). Zymosan injection (i.pl.) upregulated GMCSF (also known as $C s f 2$ ), $C c l 17, I l 1 b$, and $T n f$ mRNA expression (by quantitative PCR [qPCR]) in WT mice (Figure 1C) (23). As some indication of the mechanism governing the TNF dependence, in the absence of TNF, the increased expression of GMCSF and Illb mRNA seen following zymosan injection in WT mice was reduced (Figure 1C). Ccl17 expression levels were lower in the saline-injected footpad of $\mathrm{Tnf}^{\prime-}$ versus WT mice (Figure 1C); following i.pl. zymosan injection, there was an approximately 2-fold increase in C Cl17 expression in the footpads of both $\mathrm{Tnf}^{\prime-}$ and WT mice (Figure 1C), thereby maintaining the difference in $C c 117$ expression levels seen between the strains in the steady state.

$\mathrm{Tnf}^{\prime-}$ mice also developed significantly less zymosan-induced arthritis (ZIA) pain and disease (Figure 2A) and the increased GMCSF, $C c l 17$, and $I l 1 b$ mRNA expression in the arthritic joints was also significantly reduced in the absence of TNF (Figure 2B), noting that, unlike for the footpad, GMCSF mRNA expression could not be detected in saline-injected joints from either WT or $\mathrm{Tnf}^{\prime-}$ mice and that there was no difference in $C c 117$ mRNA expression in the saline-injected joints from $\mathrm{Tnf}^{\prime-}$ versus WT mice. Interestingly, prophylactic (Figure 3A), but not therapeutic (Figure 3B), treatment with anti-TNF mAb was able to ameliorate pain and disease (histology), whereas both prophylactic (Figure $3 \mathrm{~A}$ ) and therapeutic (Figure 3B) treatment with anti-GM-CSF mAb was able to do so. Therapeutic treatment with anti-CCL17 mAb was also able to ameliorate pain and disease (Figure 3B), paralleling the prior findings seen using $C C l 17^{E / E}$ mice (23).

Thus, in the zymosan-induced models studied, the effects of TNF deletion on inflammatory pain, as well as on arthritic pain and disease, parallel those of GM-CSF and CCL17 deletion/neutralization (23), and indicate a possible nexus. TNF blockade is able to prevent the onset of arthritic pain and disease but, in contrast, once pain is established, TNF blockade is unable to reverse the pain and disease, unlike GM-CSF or CCL17 blockade. In both models, zymosan increased GMCSF and Ccl17 mRNA expression, with the degree of TNF dependence being higher in the joint than in the footpad; unlike in the footpad, in the joint no GMCSF mRNA was detected basally - the basal GMCSF mRNA levels in the respective tissues could contribute to the degree of TNF dependence of the increased Ccl17 mRNA expression following zymosan administration. 
A

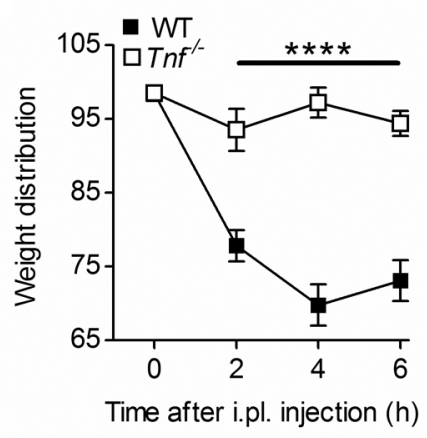

B

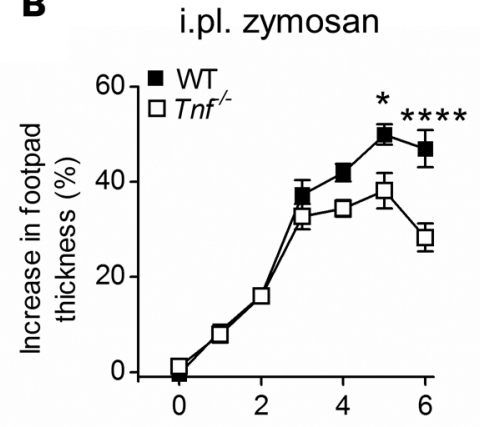

Time after zymosan injection (h)

\section{C i.pl. zymosan}
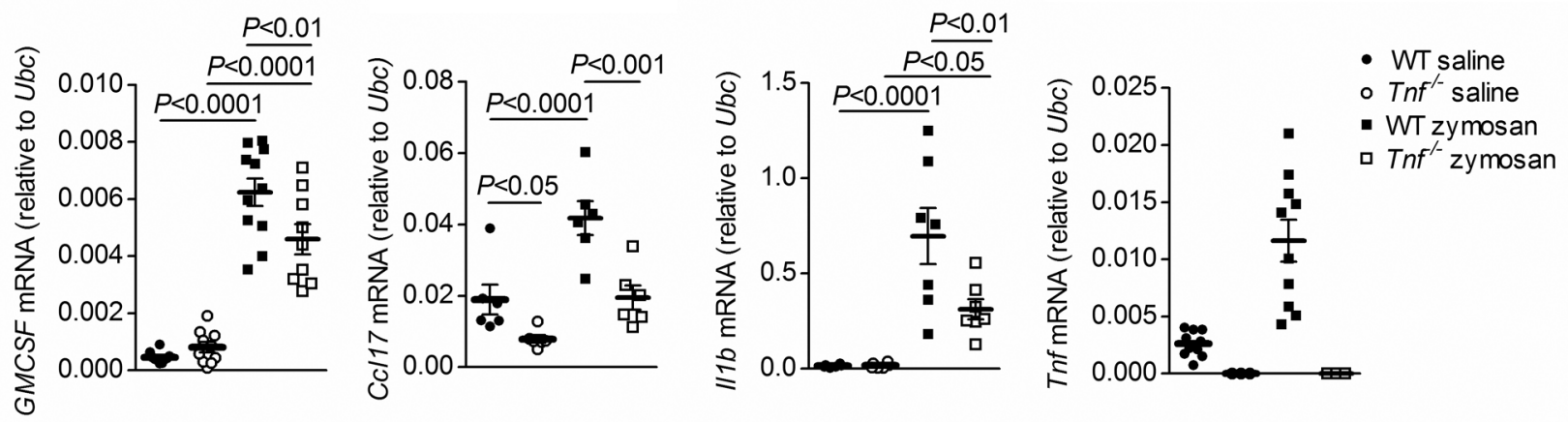

Figure 1. TNF is required for zymosan-induced inflammatory pain. (A-C) WT and Tnf $f^{-1-}$ mice received an intraplantar (i.pl.) injection of zymosan, and (A) pain (incapacitance meter [ratio of weight bearing on injected relative to noninjected hindlimb]; a value < 100 indicates pain), (B) swelling, and (C) footpad mRNA expression (6 hours zymosan or saline) were measured ( $n=6-10$ mice/group). Results are shown as mean \pm SEM. $P$ values were obtained using a 2-way ANOVA. ${ }^{*} P<0.05,{ }^{* * * *} P<0.0001$, WT versus $T \mathrm{Tnf}^{-/}$mice.

We previously showed that i.pl. CFA-induced inflammatory pain, but not footpad swelling, was also both GM-CSF and CCL17 dependent (23). Using $\operatorname{Tnf}^{\prime-}$ mice, we show that pain is also TNF dependent in this model (Supplemental Figure 1A; supplemental material available online with this article; https://doi.org/10.1172/jci.insight.99249DS1) and there is some reduction in the degree of footpad swelling (thickness) in the absence of TNF (Supplemental Figure 1B).

GM-CSF-driven inflammatory pain is TNF dependent. We have previously demonstrated that exogenous GM-CSF-driven inflammatory pain and exogenous GM-CSF-driven arthritic pain and disease are dependent on CCL17, and that Ccl17 mRNA expression is highly induced by GM-CSF in these models and in monocytes/macrophages in vitro (23). Tnf mRNA expression was also upregulated in the footpad of mice following i.pl. GM-CSF injection and in the joints of mice given i.a. methylated BSA (mBSA) and s.c. GMCSF, i.e., in the so-called mBSA/GM-CSF arthritis model (23).

We firstly explored whether GM-CSF-induced inflammatory pain is dependent on TNF. Following i.pl. GM-CSF injection, WT mice, as before (23), developed pain, whereas $\mathrm{Tnf}^{\prime-}$ mice did not (Figure 4A). As part of the analysis of the underlying mechanisms, we also monitored cytokine gene expression in the footpad both before and after GM-CSF injection. In the naive footpad, similar to the saline-injected footpad (Figure 1C), Tnf ${ }^{\prime-}$ mice had significantly lower Ccl17 mRNA expression, but similar GMCSF and Illb mRNA expression, compared with WT mice (Supplemental Figure 2). Upon i.pl. GM-CSF injection, we found previously that Ccl17, GMCSF, and Il1b mRNA expression in the plantar tissue were all upregulated in WT mice (23). For Ccl17 mRNA there was an approximately 8-fold increase, while in $T n f^{\prime-}$ mice this relative difference was maintained after GM-CSF administration (Supplemental Figure 2); upregulation of $G M C S F$, but not of $I l 1 b$, mRNA expression following i.pl. GM-CSF was dependent on TNF.

As for the $T n f^{\prime-}$ mouse, i.pl. anti-TNF mAb, given at the same time as i.pl. GM-CSF, also blocked the rapid pain induction (Figure 4B). Upon TNF blockade the increased GMCSF mRNA expression 
A

i.a. zymosan
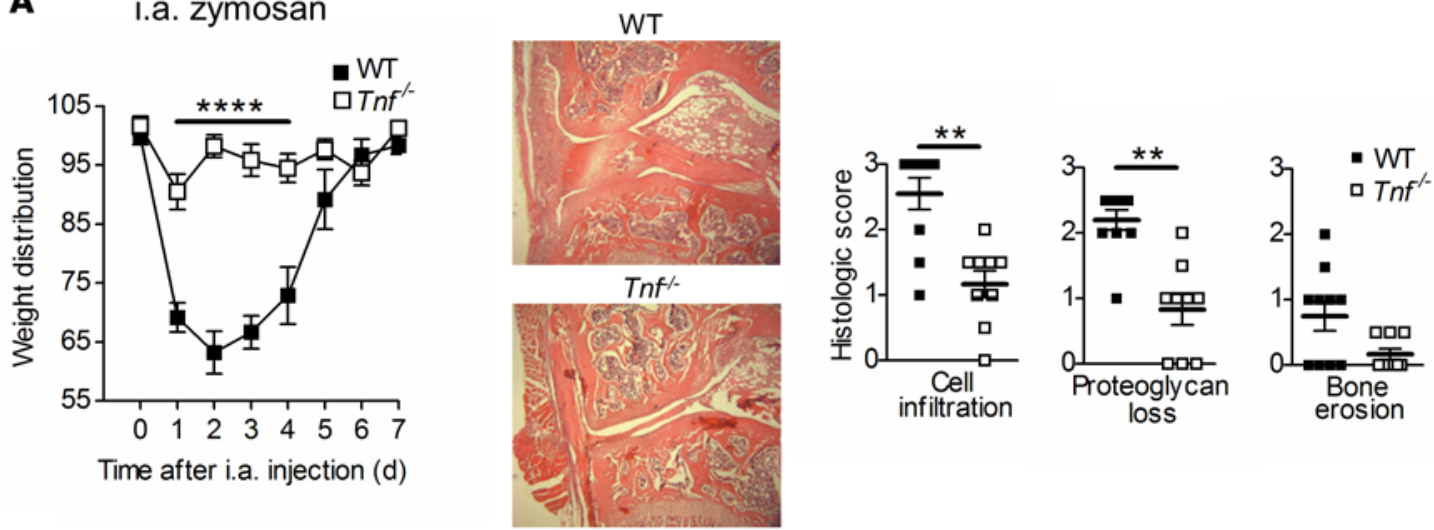

B

i.a. zymosan
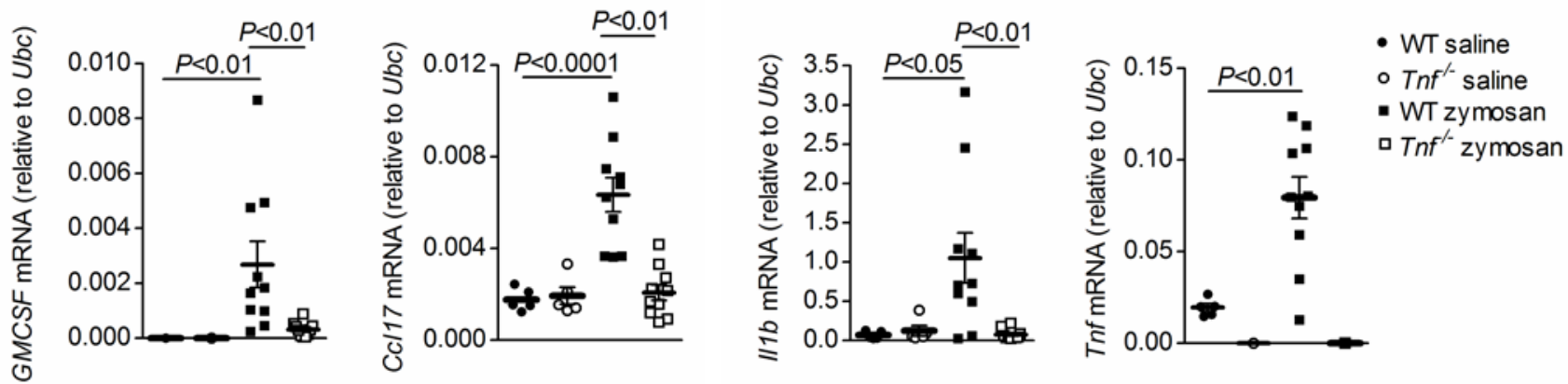

Figure 2. TNF is required for zymosan-induced arthritic pain and optimal disease. (A and B) WT and Tnf $^{-/-}$mice received an intra-articular (i.a.) injection of zymosan; (A) pain and arthritis (histology, day 7) and (B) joint mRNA expression (day 7 zymosan or saline) were measured ( $n=5-10$ mice/group). For histology images, original magnification $\times 60$. Results are shown as mean $\pm \mathrm{SEM}$. $P$ values were obtained using a 2-way ANOVA test for pain (weight distribution) readings $(\mathbf{A})$ and gene expression $(\mathbf{B})$, and Mann-Whitney $U$ test for histology $(\mathbf{A}) .{ }^{* *} P<0.01,{ }^{* * * *} P<0.0001$, WT versus $\operatorname{Tnf}^{-/-}$mice.

was reduced and there was a partial reduction in C Cl17 mRNA expression; there was, however, no effect on $I l 1 b$ or Tnf mRNA expression (data not shown).

These data indicate that GM-CSF-driven inflammatory pain, in addition to requiring CCL17 (23), also requires TNF which is also necessary for the increased GMCSF mRNA expression observed upon i.pl. GM-CSF administration. Naive-footpad Ccl17 mRNA levels appear to be partially dependent on TNF; however, the similar fold increase in $C c 117$ mRNA expression seen following i.pl. GM-CSF administration in both WT and $T n f^{\prime-}$ mice indicates that GM-CSF is able to increase $C c l 17$ mRNA expression in a TNFindependent manner.

Tnf ${ }^{\prime-}$ mice are resistant to GM-CSF-driven arthritic pain and disease. To explore whether TNF is also required for GM-CSF-driven arthritic pain and disease development, we again took advantage of our monoarticular mBSA/GM-CSF arthritis model (i.a. mBSA at day 0, s.c. GM-CSF days 0-2) (23, 25). As for the gene-deficient $C_{C l 1} 7^{E / E}$ mice (23), $T n f^{\prime-}$ mice did not develop mBSA/GM-CSF-induced arthritic pain or optimal disease (Figure 4C).

As a continuation of the mechanistic analysis we next determined whether joint $C c l 17$ mRNA expression is dependent on TNF. Naive joints from $T n f^{\prime-}$ mice had similar, albeit low, $C c l 17$ mRNA expression compared with WT mice (Supplemental Figure 3). Saline-injected (i.a.) joints (saline/saline in Supplemental Figure 3) showed Ccl17 mRNA expression similar to that of naive joints in both strains. As for i.a. zymosan (Figure 2B), mBSA-injected joints (mBSA/saline in Supplemental Figure 3) increased Ccl17 mRNA expression more in WT mice compared with $T n f^{\prime-}$ mice (day 7 ; i.e., at termination) (Supplemental Figure 3). The addition of s.c. GM-CSF led to a further increase in Ccl17 mRNA expression in the mBSA-injected joints (mBSA/GM-CSF in Supplemental Figure 3) that, however, was similar in both strains, whereas it had no effect in the salineinjected joints (saline/GM-CSF in Supplemental Figure 3). Similar to the Figure 2B data, GMCSF mRNA expression could not be detected in naive, saline-, or mBSA-injected joints from WT and $T n f^{\prime-}$ mice, with or without the addition of s.c. GM-CSF (data not shown). 
A

i.a. zymosan

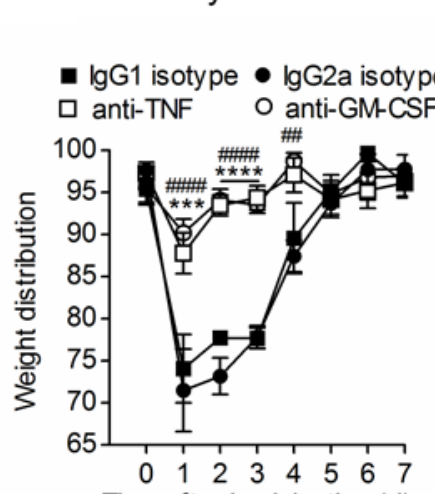

Time after i.a. injection (d)

B

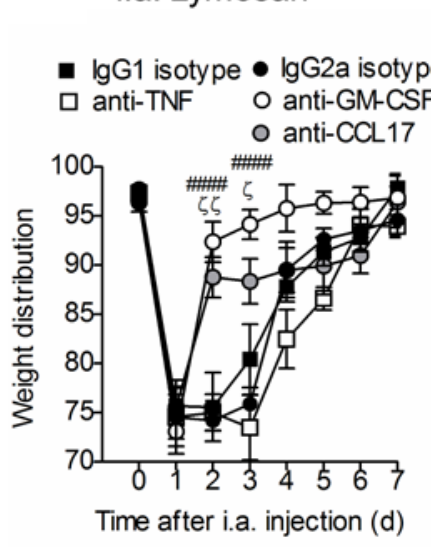

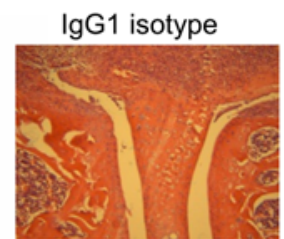
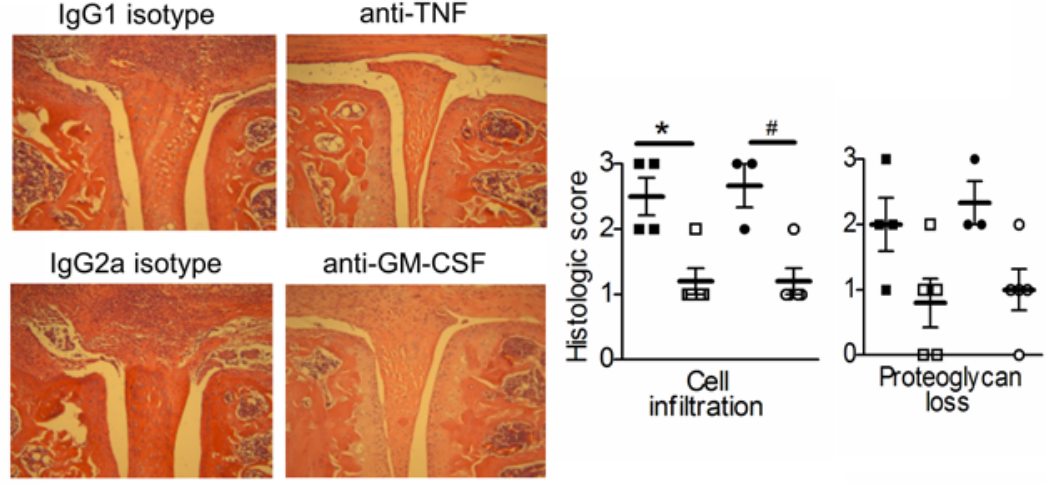

- $\lg \mathrm{G} 1$ isotype

口 anti-TNF

- $\lg \mathrm{G} 2 \mathrm{a}$ isotype

- anti-GM-CSF

3

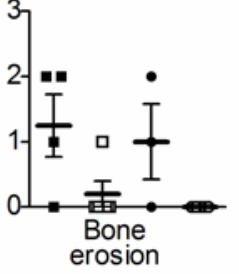

i.a. zymosan
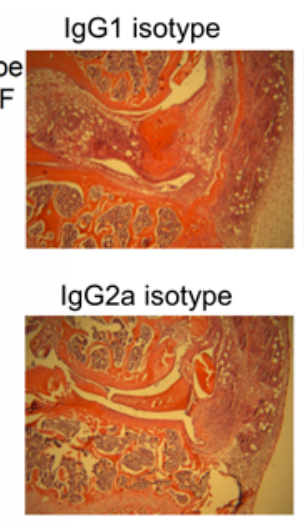

anti-TNF

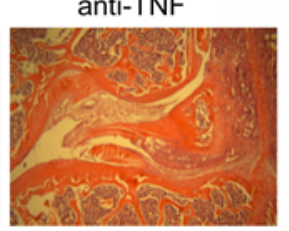

anti-GM-CSF

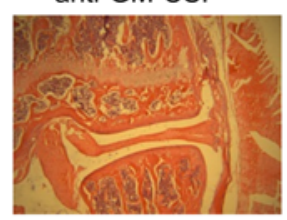

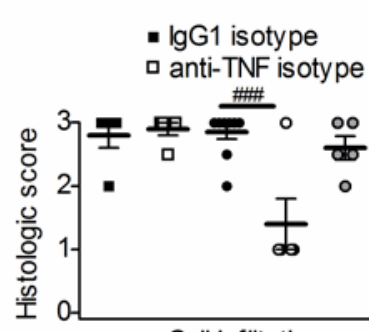

Cell infiltation

anti-CCL17

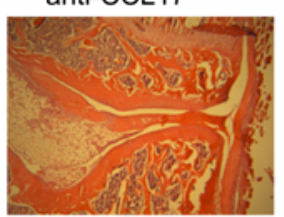

- lgG2a isotype

- anti-GM-CSF

- anti-CCL17 \#

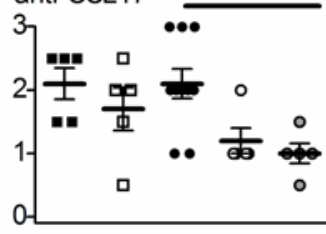

Proteoglycan loss

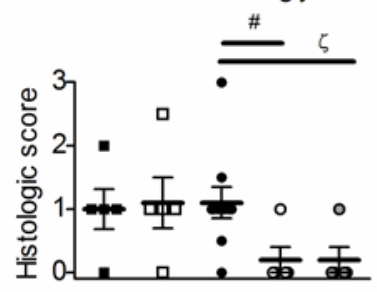

Bone erosion

Figure 3. TNF is not required for the maintenance of zymosan-induced arthritic pain and disease. (A and B) WT mice received an intra-articular (i.a.) injection of zymosan and anti-TNF mAb, anti-granulocyte macrophage-colony stimulating factor (anti-GM-CSF) mAb, IgG1 or lgG2a isotype control mAbs (150 $\mu$ g i.p.), either (A) prophylactically (on days -2 and 0$)(n=4-5$ mice/group) or (B) therapeutically (day 1$)$ ( $n=5-10$ mice/group), and pain and arthritis (histology, day 7) were measured. Mice were also treated (B) therapeutically with anti-CCL17 mAb (150 $\mu$ g i.p.) (day 1) ( $n=5$ mice/group). For histology images, original magnification $\times 60$. Results are shown as mean $\pm \mathrm{SEM}$. $P$ values were obtained using a 2-way ANOVA test for pain (weight distribution)

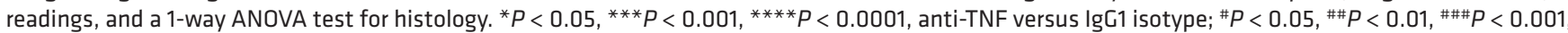
$\# \# \# P<0.0001$, anti-GM-CSF versus IgG2a isotype; ${ }^{\complement} P<0.05,{ }^{5} P<0.01$, anti-CCL17 versus IgG2a isotype.

These data indicate that GM-CSF-driven arthritic pain and disease require TNF. The increased Ccl17 mRNA expression in mBSA-injected joints is partially dependent on TNF, similar to the naive footpad; however, the further increase in C $c 117$ mRNA expression seen in mBSA-injected joints following s.c. GM-CSF is independent of TNF.

GM-CSF-induced CCL17 expression is not TNF dependent in murine monocytes/macrophages. Having shown that GM-CSF-driven inflammatory pain and GM-CSF-driven arthritic pain and disease were both TNF and CCL17 (23) dependent, yet paradoxically the GM-CSF-enhanced Ccl17 mRNA expression in both the footpad (Supplemental Figure 2) and the joint (Supplemental Figure 3) were not, we next determined whether TNF was required for CCL17 production by GM-CSF-treated monocytes/macrophages in vitro to assist in the mechanistic analysis. We have previously shown that GMCSF-stimulated murine macrophage and human monocyte populations upregulate both Ccl17 and Tnf mRNA expression (23). GM-CSF treatment of thioglycolate-elicited peritoneal macrophages from WT or $\mathrm{Tnf}^{\prime-}$ mice in vitro resulted in similar CCL17 secretion (Supplemental Figure 4A) and anti-TNF $\mathrm{mAb}$ did not reduce such induced secretion from WT macrophages (Supplemental Figure 4B). Similarly, anti-TNF mAb treatment did not affect CCL17 secretion from GM-CSF-stimulated $\mathrm{CD} 115^{+} \mathrm{CD} 11 \mathrm{~b}^{+}$murine bone marrow monocytes (Supplemental Figure 4C); TNF itself did not lead to $C c l 17$ induction in these populations (data not shown). 
A

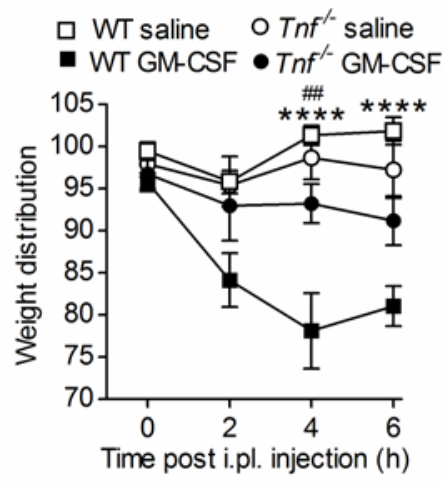

B

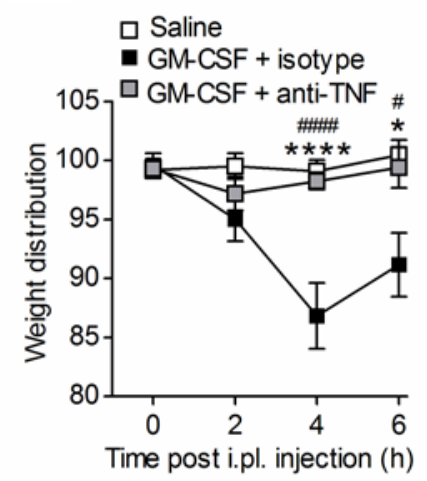

\section{C $\quad \mathrm{mBSA} / \mathrm{GM}-\mathrm{CSF}$ arthritis}
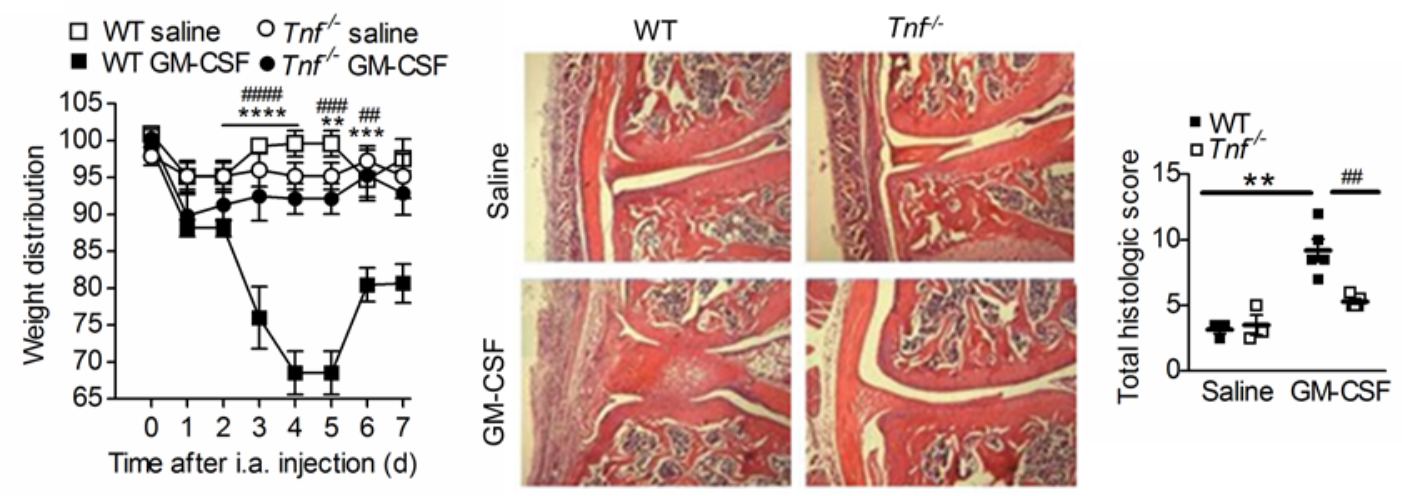

Figure 4. GM-CSF-driven inflammatory pain, as well as GM-CSF-driven arthritic pain and disease, are TNF dependent. (A and B) Intraplantar (i.pl.) injection of granulocyte macrophage-colony stimulating factor (CM-CSF) (20 ng) or saline in (A) WT and Tnf ${ }^{-1-}$ mice $(n=4-6$ mice/group) and in (B) WT mice treated with anti-TNF or isotype control $(2 \mu \mathrm{g} / \mathrm{paw}$ i.pl. at $t=0)(n=12$ mice/group). Pain was measured. (C) Methylated BSA (mBSA)/GM-CSF arthritis (intra-articular [i.a.] mBSA [day 0] and GM-CSF or saline s.c. [days 0-2]) was induced in WT and Tnf ${ }^{-1-}$ mice. Pain and arthritis (histology, day 7) were measured ( $n=4-6$ mice/group). Original magnification, $\times 60$. Results are shown as mean $\pm \mathrm{SEM} . P$ values were obtained using a 2 -way ANOVA. ${ }^{*} P<0.05,{ }^{*} P$

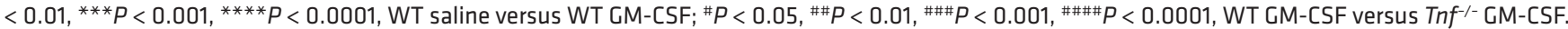

These data suggest that GM-CSF-induced CCL17 production in these murine monocyte/macrophage populations is not dependent on autocrine/paracrine TNF production, consistent with our in vivo findings above in the GM-CSF-driven models.

Prophylactic, but not therapeutic, treatment with anti-TNF mAb ameliorates GM-CSF-driven arthritic pain and disease. Our GM-CSF-driven inflammatory and arthritic models are both TNF dependent (Figure 4), and CCL17 dependent (23), yet GM-CSF-induced CCL17 formation in these models and in murine monocytes/macrophages does not require TNF even though GM-CSF can increase its mRNA expression. We therefore tested at what stage TNF might be required in the mBSA/GM-CSF model using timed neutralizing anti-TNF $\mathrm{mAb}$ administration. As for the ZIA model above, anti-TNF mAb, given prophylactically (days -2 and 0 , day 0 being the time of arthritis induction), was able to prevent onset of pain and disease (Figure 5A); however, therapeutic anti-TNF mAb treatment (on day 4, once pain was evident) had no effect on the pain and disease progression (Figure 5B). Therapeutic treatment with anti-CCL17 mAb (day 4), on the other hand, ameliorated the pain and disease (Figure 5B).

These data indicate that TNF is required for GM-CSF to be able to induce detectable pain and enhance disease in the mBSA-injected joint; however, once pain is established TNF is no longer required. In contrast, CCL17 is needed for the ongoing maintenance of pain and disease in this model.

GM-CSF is required for TNF-driven inflammatory pain. Having shown above that the initiation of GM-CSFdriven inflammatory and arthritic pain are dependent on TNF, we next determined whether, inversely, TNFdriven inflammatory pain requires GM-CSF. TNF (i.pl.) has been shown to induce pain that is cyclooxygenase dependent $(21,22)$. As for i.pl. GM-CSF-induced pain (23), we initially confirmed that i.pl. TNF-induced pain 
A

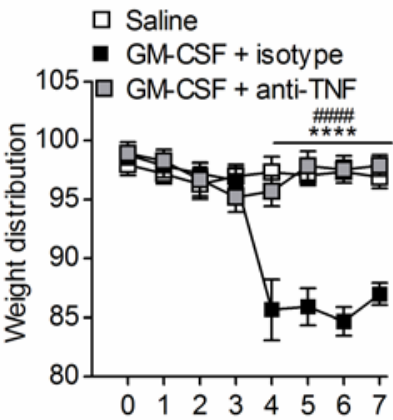

Time after i.a. injection (d)

B

mBSA/GM-CSF arthritis

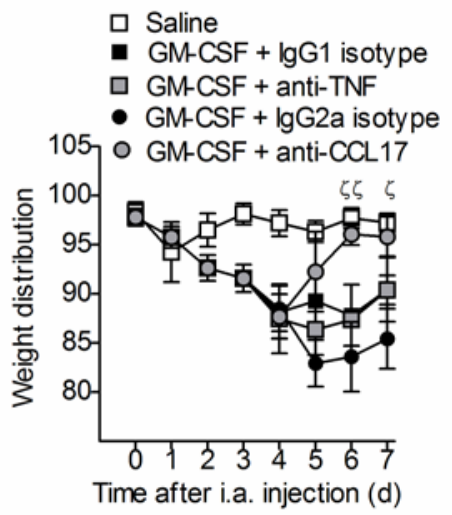

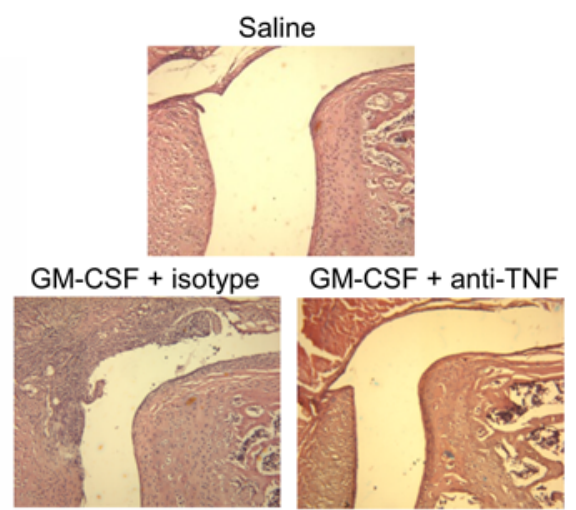
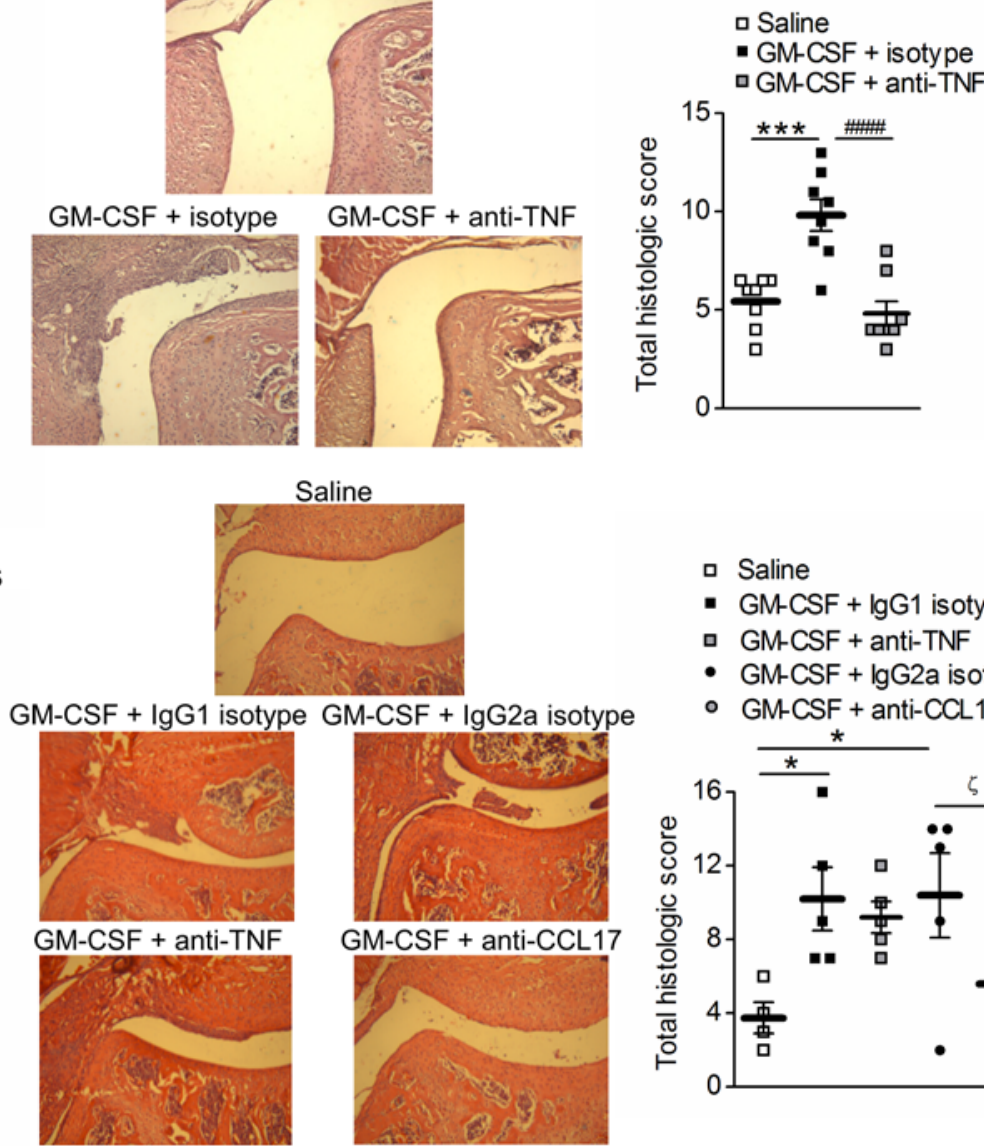

- Saline

- GM-CSF + lgG1 isotype

- GM-CSF + anti-TNF

- GM-CSF + lgG2a isotype

- GM-CSF + anti-CCL17

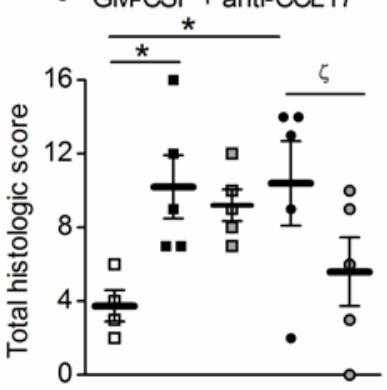

Figure 5. Prophylactic, but not therapeutic, treatment with anti-TNF mAb ameliorates GM-CSF-driven arthritic pain and disease. (A and B) Methylated BSA (mBSA)/granulocyte macrophage-colony stimulating factor (GM-CSF) arthritis (intra-articular [i.a.] mBSA [day 0] and GM-CSF or saline s.c. [days 0-2]) was induced in WT mice treated with anti-TNF mAb or IgG1 isotype control (150 $\mu$ g i.p.) (A) prophylactically (on days -2 and 0 ) ( $n=8-12$ mice/group) and (B) therapeutically (day 4) ( $n=5$ mice/group). Mice were also treated (B) therapeutically with anti-CCL17 mAb or IgG2a isotype control (150 $\mu$ g i.p.) (day 4 ) ( $n=4-5$ mice/group). Pain (incapacitance meter) and arthritis (histology) were measured. Original magnification, $\times 125$. Results are shown as mean \pm SEM. $P$ values were obtained using a 2-way ANOVA test for pain (weight distribution) readings and a Mann-Whitney $U$ test or 1-way ANOVA for histology quantification. ${ }^{*} P<$

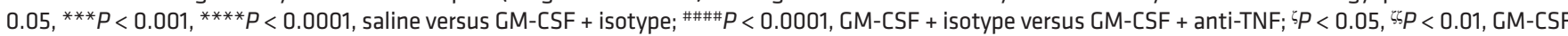
+ isotype versus GM-CSF + anti-CCL17.

could be reversed by the cyclooxygenase inhibitor, indomethacin (Figure 6A); the COX-2 inhibitor, SC58125, also blocked TNF-driven pain, whereas the sympathetic amine inhibitor, guanethidine, did not (Figure 6B).

$\mathrm{GMCSF}^{-1-}$ mice were resistant to i.pl. TNF-driven pain development (Figure 6C). TNF (i.pl.) led to the upregulation of GMCSF, Ccl17, Il1b, and Tnf mRNA expression (measured at 4 hours) in WT mice (Supplemental Figure 5). As before (23), Ccl17 mRNA expression was barely detectable in the saline injected footpad of $\mathrm{GMCSF}^{-1-}$ mice and was not significantly increased by i.pl. TNF injection in these mice (Supplemental Figure 5); $I l 1 b$ and $T n f$ mRNA expression, on the other hand, was similarly increased in $G M C S F^{-/}$mice and WT mice following i.pl. TNF injection. Blockade of GM-CSF, using a neutralizing mAb given i.pl. at the same time as i.pl. TNF, also inhibited TNF-induced pain (Figure 6D) and Ccl17 mRNA expression but not that of $I l 1 b$ or $\operatorname{Tnf}$ mRNA (data not shown).

These data indicate that in the footpad GM-CSF is required for TNF-driven inflammatory pain and elevated $C$ cl17 mRNA expression.

GM-CSF is required for TNF-driven arthritic pain and disease. To assess whether GM-CSF is also required for TNF-driven arthritic pain and disease, and to enable us potentially to determine pathways downstream of TNF action in arthritic pain and disease, we substituted TNF for GM-CSF to establish an mBSA/TNF arthritis model. In this monoarticular model (i.a. mBSA at day 0, s.c. TNF days 0-2) pain was evident at 6 hours after mBSA i.a. injection, which is much earlier than in either the mBSA/ 
A

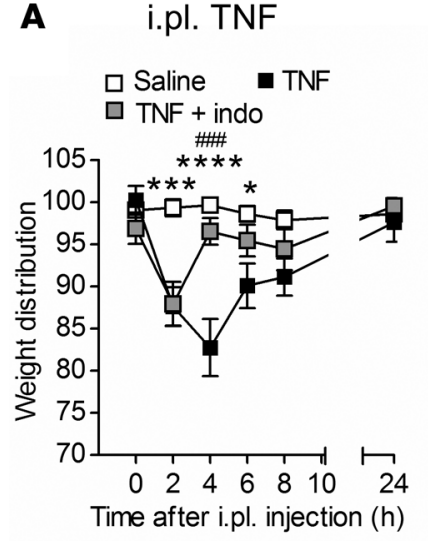

C

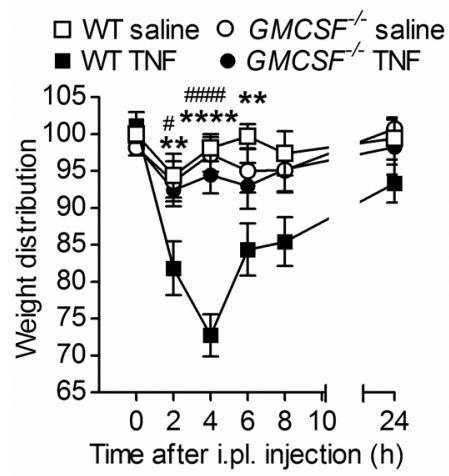

B

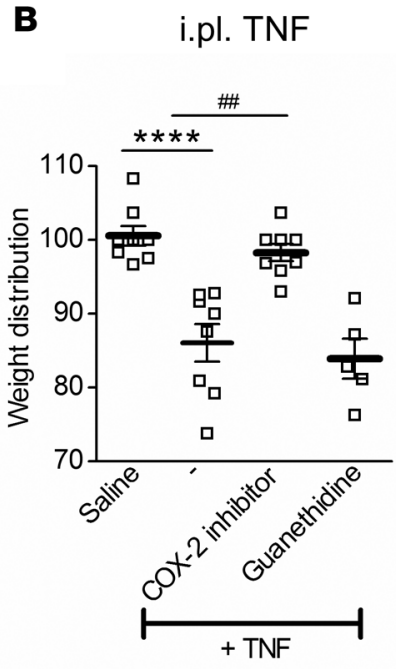

D i.pl. TNF

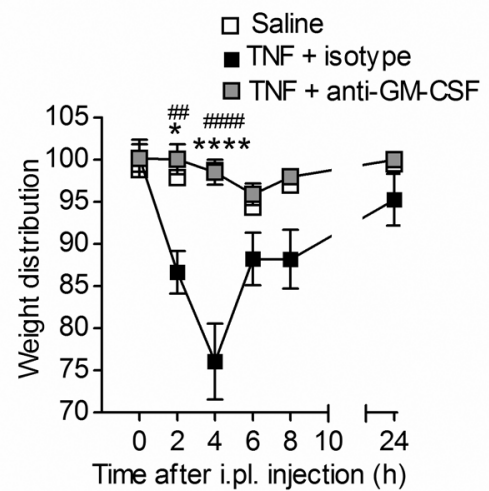

Figure 6. GM-CSF is required for TNF-driven inflammatory pain. (A-D) Intraplantar (i.pl.) injection of TNF (20 ng) or saline in (A) WT mice treated with/without indomethacin $(12.5 \mu \mathrm{g} /$ paw i.pl. at 2 hours) ( $n=10$ mice/group), (B) WT mice treated with/without the COX-2 inhibitor, SC58125 (5 mg/kg i.p. at $t=-30$ minutes), or guanethidine sulfate $(25 \mathrm{mg} / \mathrm{kg}$ s.c. at -60 minutes) for 4 hours ( $n=5-8$ mice/group), (C) WT and $\mathrm{GMCSF}^{-/}$mice ( $n=5-10$ mice/group), and (D) WT mice treated with anti-GM-CSF mAb or isotype control $(2 \mu \mathrm{g} /$ paw i.pl. at $t=0)$ ( $n=5$ mice/group). Pain (incapacitance meter) was measured. Results are shown as mean \pm SEM. $P$ values were obtained using a 1-way (B) or 2-way (A, C, and D) ANOVA test. ${ }^{*} P<0.05,{ }^{* *} P<0.01,{ }^{* *} P<0.001,{ }^{* * *} P<0.0001$, WT saline versus WT TNF or TNF + isotype; ${ }^{\#} P<0.05,{ }^{\# \# P<0.01, ~ \# \# \#<}$ 0.001 , \#\#\#\# $P<0.0001$, TNF versus TNF + indo or TNF + COX-2 inhibitor; WT TNF versus GMCSF-1- TNF; TNF + isotype vs. TNF + anti-GM-CSF. GM-CSF, granulocyte macrophage-colony stimulating factor.

GM-CSF (Figure 4C) or mBSA/CCL17 models (23), and lasted only until day 4 (Figure 7A). As for the mBSA/GM-CSF and mBSA/CCL17 models (23), indomethacin treatment from 6 hours onwards was able to reverse this pain within 2 hours (Figure 7A). There was also a significant reduction in disease at day 7, although not to the level of that seen in $\mathrm{mBSA} /$ saline mice. Similar to these other models (23), our mBSA/TNF model was also active in $R a g 1^{-1-}$ mice (Supplemental Figure 6), indicating $\mathrm{T}$ and $\mathrm{B}$ lymphocyte independence.

As for i.pl. TNF-induced pain, $G M C S F^{-1-}$ mice did not develop mBSA/TNF-induced arthritic pain onset or disease (Figure 7B) and prophylactic treatment with an anti-GM-CSF mAb prevented arthritic pain and disease development (Figure 8A). Anti-GM-CSF mAb treatment reduced the Ccl17 mRNA expression in the mBSA-injected joints from mice receiving s.c. TNF to that seen in saline-injected joints at day 7 , and below that seen in mBSA-injected joints of mice receiving s.c. saline (Supplemental Figure 7), thus confirming the GM-CSF dependence of Ccl17 expression. Therapeutic treatment with an anti-GM-CSF mAb (day 2) was also able to ameliorate the mBSA/TNF-driven arthritic pain and disease (Figure 8B).

Thus, GM-CSF is required for TNF to be able to induce detectable pain and enhance disease in the mBSA-injected joint; timed neutralizing $\mathrm{mAb}$ administration indicated that it is also needed for the ongoing maintenance of pain and disease in the mBSA/TNF model. In this model GM-CSF is absolutely required for any increase in TNF-driven $C c 117$ mRNA expression.

IRF4 and CCL17 are also required for TNF-driven inflammatory pain and for TNF-driven arthritic pain and disease. Seeing that TNF-driven inflammatory pain and TNF-driven arthritic pain and disease are dependent on GM-CSF (Figure 6, C and D, Figure 7B, and Figure 8, A and B) and that GM-CSFdriven inflammatory and arthritic pain are dependent on CCL17 via IRF4 (23), we next determined whether TNF-driven pain utilizes the same GM-CSF-dependent pathway as a contributing mechanism. Indeed $\mathrm{Irf}^{-{ }^{--}}$mice were resistant to i.pl. TNF-driven pain (Supplemental Figure 8A). Following i.pl. 
A mBSA/TNF arthritis

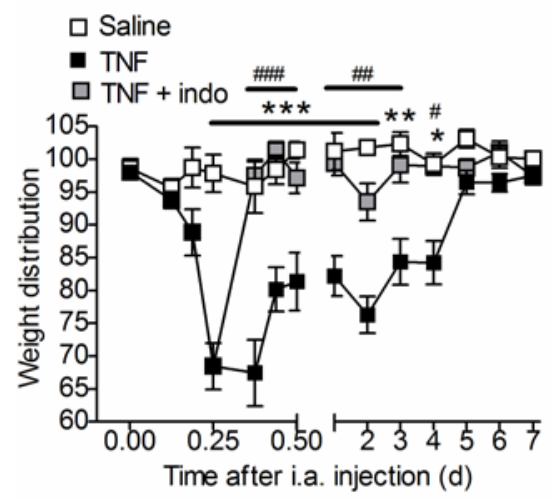

B

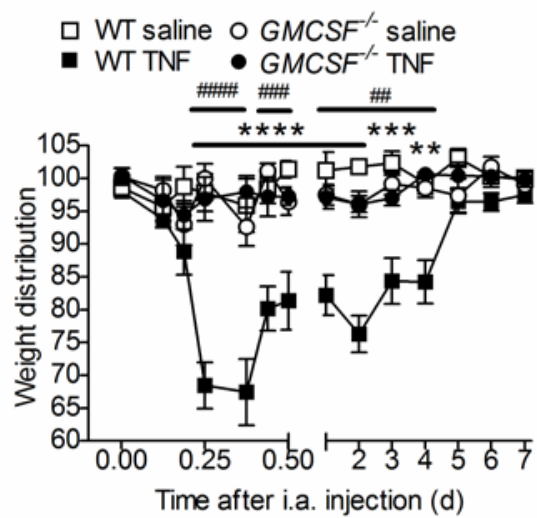

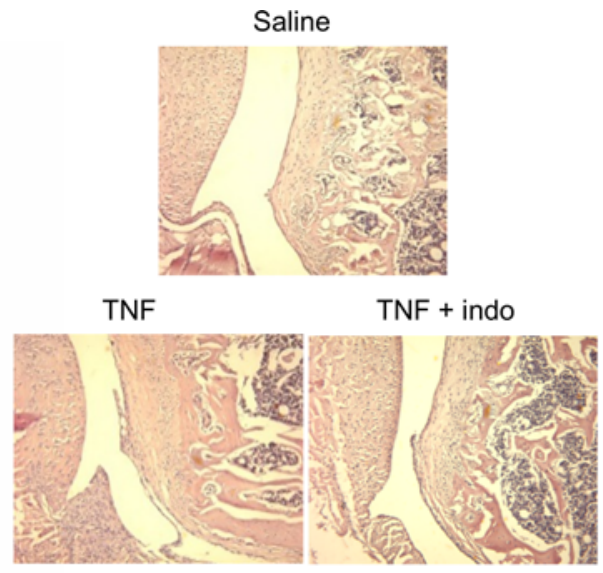

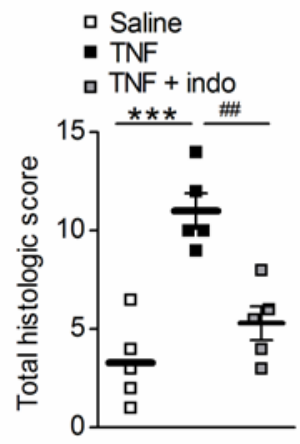

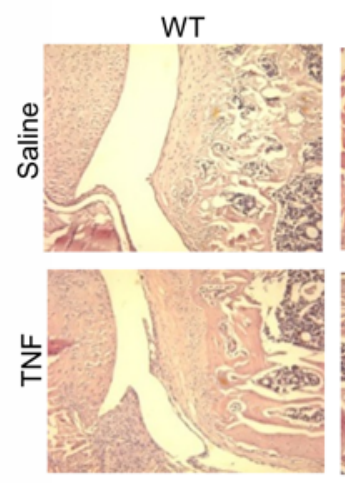

GMCSF $\%$
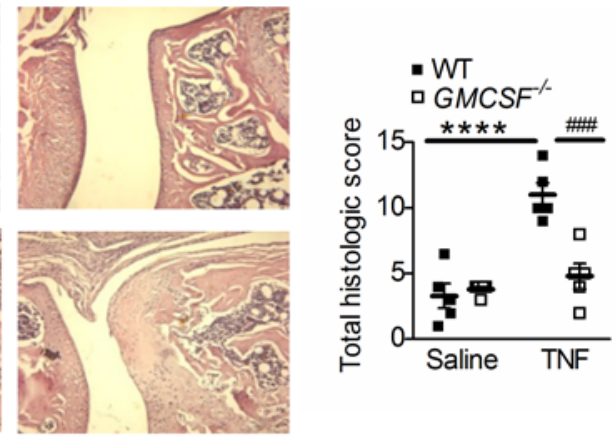

Figure 7. GM-CSF is required for TNF-driven arthritic pain and disease. (A and B) Methylated BSA (mBSA)/TNF arthritis (intra-articular [i.a.]mBSA [day 0] and TNF or saline s.c. [days 0-2]) was induced in (A) WT mice treated with/without indomethacin (1 mg/kg, i.p. from 6 hours) ( $n=7-10$ mice/group), and (B) CMCSF $^{-1-}$ mice ( $n=8-10$ mice/group). Pain and arthritis (histology) were measured. Original magnification, $\times 125$. Results are shown as mean \pm SEM. $P$ values were obtained using a 2-way ANOVA test for pain (weight distribution) readings, a 1-way (A) or 2-way (B) ANOVA test for histology quantification.

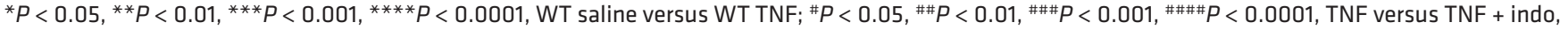
WT TNF versus $\mathrm{CMCSF}^{-/-}$TNF. GM-CSF, granulocyte macrophage-colony stimulating factor.

TNF injection, the increased Ccl17 mRNA expression noted in WT mice (Supplemental Figure 5) was significantly reduced in the plantar skin of Irft ${ }^{--}$mice, whereas the increased GMCSF, Illb, and Tnf

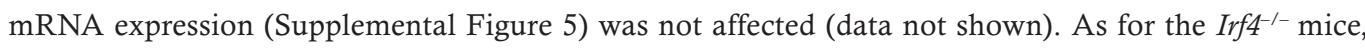
CCl1 $7^{E / E}$ mice were also resistant to i.pl. TNF-driven pain (Supplemental Figure 8B) and no differences in GMCSF, Illb, and Tnf mRNA expression in their plantar skin were seen compared with WT mice (data not shown).

To assess whether IRF4 and CCL17 were also required for TNF-driven arthritic pain and disease, the mBSA/TNF arthritis model was once again utilized. Both Irf4 ${ }^{-1}$ mice (Supplemental Figure 8C) and $\mathrm{CCl1}^{\mathrm{E} / \mathrm{E}}$ mice (Figure 9A) mice were resistant to arthritic pain and disease development. As for the antiGM-CSF mAb (Figure 8B), therapeutic anti-CCL17 mAb treatment (day 2) ameliorated the pain and disease (Figure 9B).

These findings indicate that TNF-driven inflammatory pain and TNF-driven arthritic pain and disease act via IRF4 and CCL17, similar to GM-CSF-driven inflammatory and arthritic pain and disease (23).

Inhibition of JMJD3 demethylase ameliorates TNF-driven inflammatory pain, and GM-CSF-and TNF-driven arthritic pain and disease. We have previously shown that GM-CSF upregulates IRF4 mRNA expression in human monocytes by enhancing JMJD3 demethylase activity and that blockade of JMJD3 activity inhibits GM-CSF-driven inflammatory pain (23). To determine whether the same mechanism is true for TNF-driven pain, particularly given its dependence on GM-CSF, IRF4, and CCL17, WT mice were pretreated i.p. with the JMJD3 inhibitor, GSK-J4, or vehicle 30 minutes prior to i.pl. TNF injection. 
A

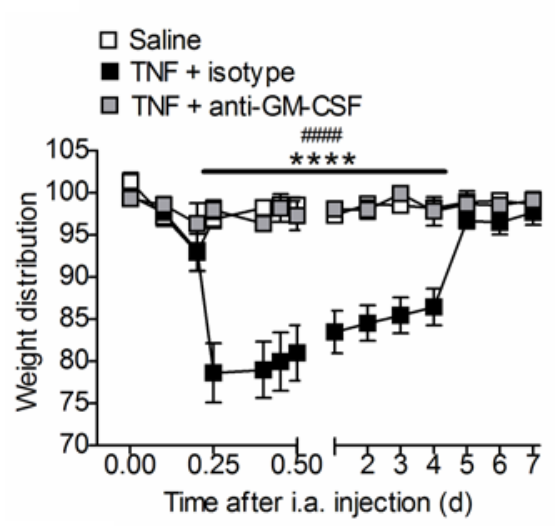

B

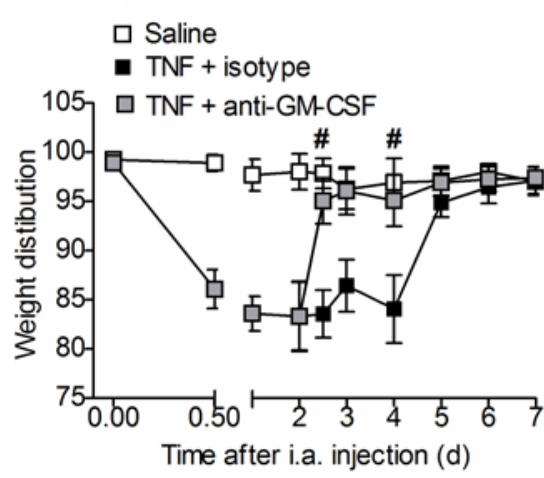

Time after i.a. injection (d)
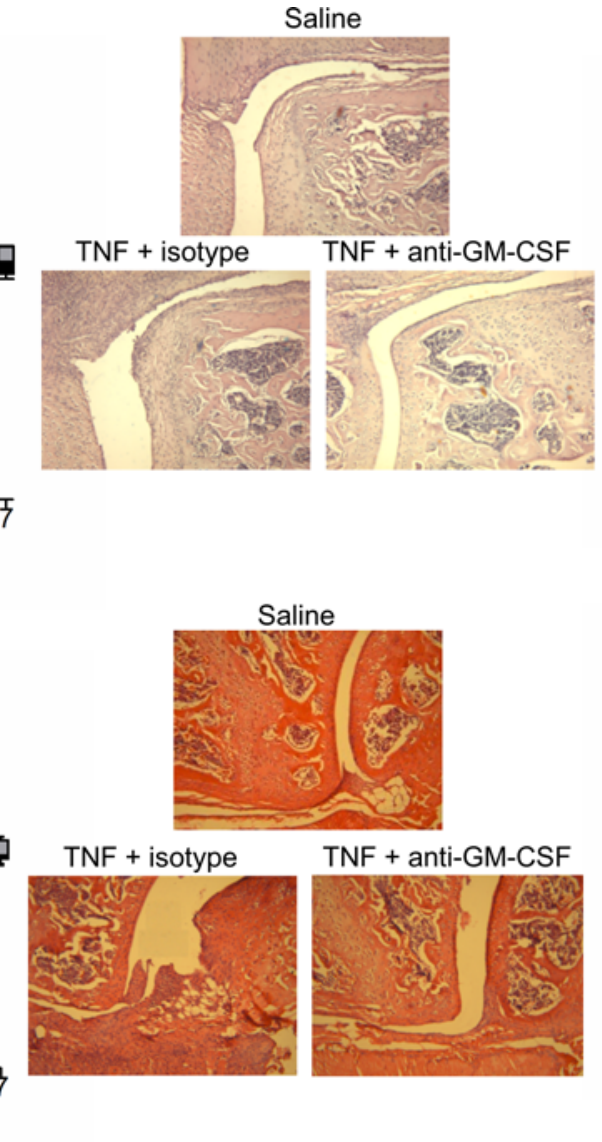
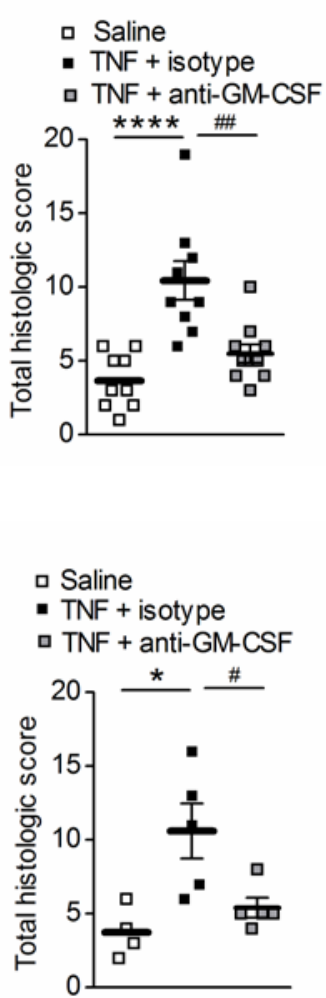

Figure 8. Prophylactic and therapeutic treatment with anti-GM-CSF mAb ameliorates TNF-driven arthritic pain and disease. (A and B) Methylated BSA (mBSA)/TNF arthritis (intra-articular [i.a.] mBSA [day 0] and TNF or saline s.c. [days 0-2]) was induced in (A) WT mice treated prophylactically with anti-granulocyte macrophage-colony stimulating factor (anti-GM-CSF) mAb or isotype control (150 $\mu$ i.p. on days -2 and 0 i.p.) ( $n=9-10$ mice/group) and (B) WT mice treated therapeutically with anti-GM-CSF mAb or isotype control (150 $\mu$ g i.p. on day 2 i.p.) ( $n=4-5$ mice/group). Pain and arthritis (histology) were measured. Original magnification, $\times 125$. Results are shown as mean \pm SEM. $P$ values were obtained using a 2-way ANOVA test for pain (weight distribution) readings, and a 1-way ANOVA test for histology quantification. ${ }^{*} P<0.05,{ }^{* * *} P<0.0001$, saline versus TNF + isotype; ${ }^{*} P<0.05$, ${ }^{\# \#} P<0.01$, $\# \# \# P<0.0001$, TNF + isotype versus TNF + anti-GM-CSF.

Similar to GM-CSF-driven pain, mice pretreated with GSK-J4 developed significantly less TNF-driven pain compared with mice pretreated with vehicle (Figure 10A).

We next determined whether GSK-J4 could also inhibit mBSA/GM-CSF- and/or mBSA/TNFdriven arthritic pain and disease development. Mice were treated daily with GSK-J4 (0.5 mg/kg i.p.) or vehicle from day -2 to day 2 , with mBSA/GM-CSF or mBSA/TNF arthritis being induced on day 0 . As above, mice pretreated with vehicle developed pain in the mBSA/GM-CSF (Figure 10B) and mBSA/TNF (Figure 10C) models, whereas mice treated with the JMJD3 inhibitor were resistant to such pain development. Mice pretreated with GSK-J4 also developed significantly less mBSA/GMCSF and mBSA/TNF arthritis (Figure 10D).

These data suggest that JMJD3 is required for the development of both GM-CSF- and TNF-driven inflammatory pain, as well as for GM-CSF- and TNF-driven arthritic pain and disease.

TNF is not required for CCL17-driven inflammatory pain and CCL17-driven arthritic pain and disease. We showed above that TNF-driven inflammatory pain (Supplemental Figure 8B) and mBSA/TNF arthritic pain and disease (Figure 9, A and B) require CCL17. In order to determine whether CCL17-driven inflammatory pain (23) requires TNF, $\mathrm{Tnf}^{\prime-}$ mice were injected i.pl. with CCL17. Pain in these mice was similar to WT mice receiving i.pl. CCL17 (Figure 11A). We have also established a CCL17-driven (mBSA/ CCL17) arthritis model (i.a. mBSA at day 0, s.c. CCL17 days 0-2), which enables us to explore pathways downstream of CCL17 (23). As can be seen in Figure 11B, and as for $G M C S F^{-1-}$ mice (23), Tnf $f^{\prime-}$ mice developed similar arthritic pain and disease to WT mice in this model. 
A

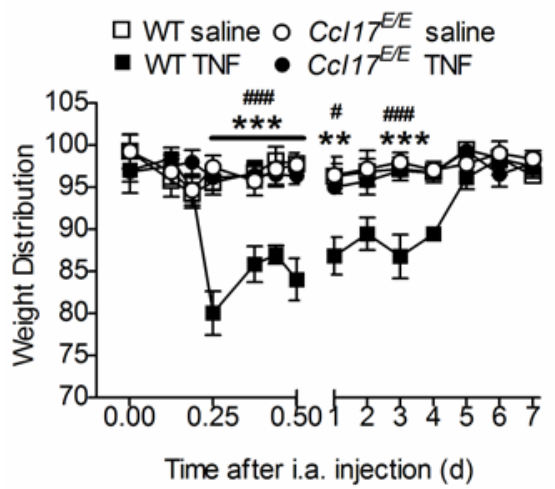

B mBSA/TNF arthritis

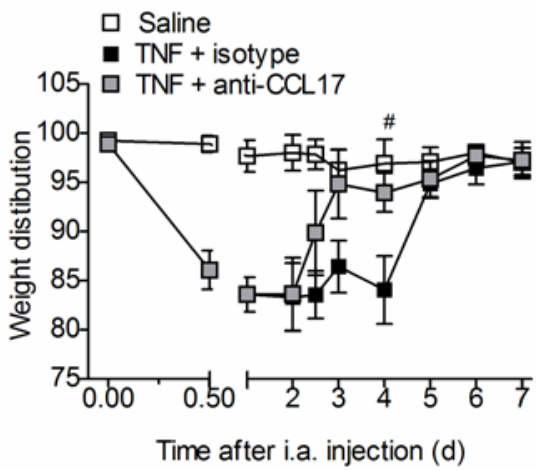

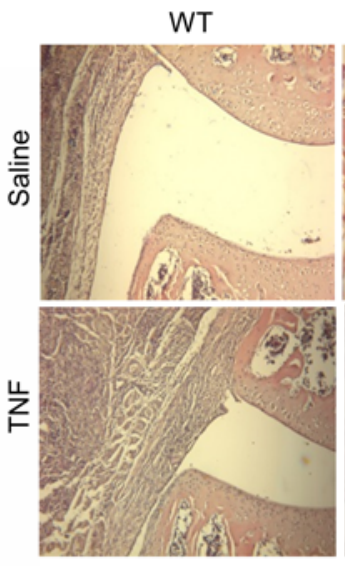

CCI17E/E
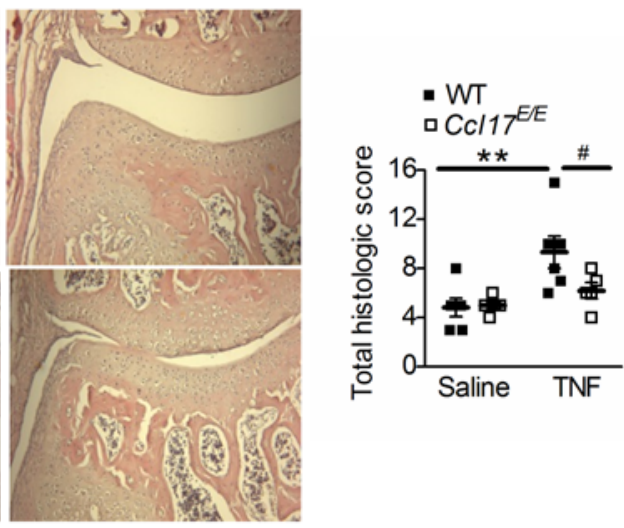

Saline
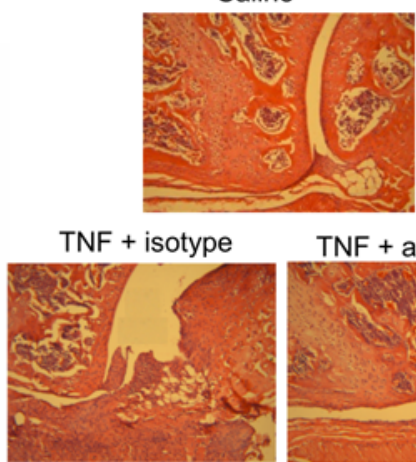

TNF + anti-CCL17
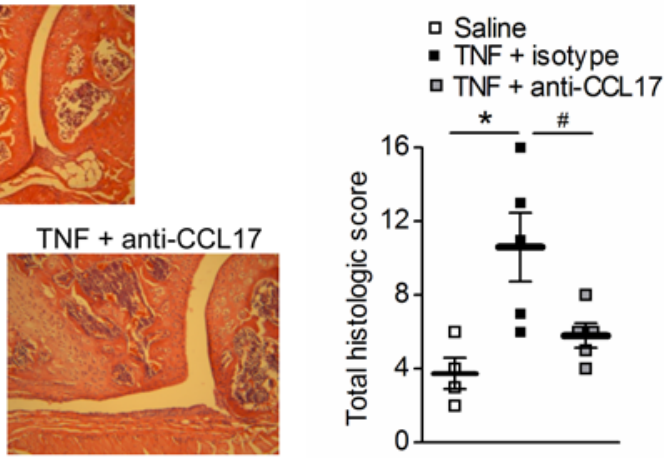

Figure 9. CCL17 is required for TNF-driven arthritic pain and disease. (A and B) Methylated BSA (mBSA)/TNF arthritis (intra-articular [i.a.] mBSA [day 0] and TNF or saline s.c. [days 0-2]) was induced in (A) WT and Cc/17 E/E mice ( $n=5-6$ mice/group) and (B) WT mice treated therapeutically with anti-CCL17 mAb or isotype control (150 $\mu$ g i.p. on day 2 i.p.) ( $n=4-5$ mice/group). Pain and arthritis (histology) were measured. Original magnification, $\times 125$. Results are shown as mean \pm SEM. $P$ values were obtained using a 2-way ANOVA test for pain and a 1-way (B) or 2-way (A) ANOVA test for histology quantification. ${ }^{*} P<0.05,{ }^{* *} P<0.01,{ }^{* * *} P<0.001$, WT saline versus WT TNF; ${ }^{\#} P<0.05,{ }^{\# \#} P<0.001$, WT TNF versus Cc/17E/E TNF; TNF + isotype versus TNF + anti-CCL17.

These data demonstrate that, like GM-CSF (23), TNF does not lie downstream of CCL17, at least in the models tested.

\section{Discussion}

We previously identified a new GM-CSF $\rightarrow \mathrm{JMJD} 3 \rightarrow \mathrm{IRF} 4 \rightarrow \mathrm{CCL} 17$ pathway that is active in monocytes/macrophages in vitro and important for the development in mice of inflammatory pain, as well as of arthritic pain and disease (23). In the present study, in support of a nexus between TNF, GM-CSF, and CCL17, initiation of zymosan-induced inflammatory pain and ZIA pain and disease are all TNF-, GM-CSF-, and CCL17-dependent (23), as is CFA-induced inflammatory pain (23); however, once established, ZIA pain and disease no longer require TNF, although there is still GM-CSF and CCL17 involvement. In addition, we showed in support of the nexus concept that TNF is required for initiation of both GM-CSF-driven inflammatory pain and GM-CSF-driven arthritic pain and disease, but not required for the maintenance of the arthritic pain and disease once established. Intriguingly, even though CCL17 is also required for the initiation, as well as for the maintenance of the pain and disease, in these GMCSF-driven models, GM-CSF-driven CCL17 expression in these models and in murine monocytes/ macrophages in vitro does not require TNF. These data suggest that TNF could also be contributing in these particular models via another interacting pathway(s) perhaps involving neutrophils (28). We also presented evidence that, on the other hand, TNF-driven inflammatory pain and TNF-driven arthritic pain and disease are dependent on GM-CSF, both for their initiation and maintenance, and as a result 
A i.pl. TNF

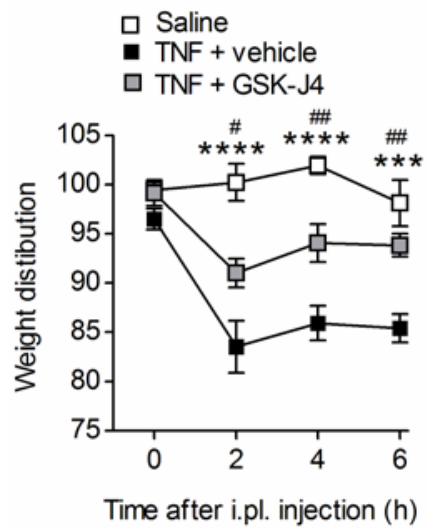

B mBSA/GM-CSF arthritis
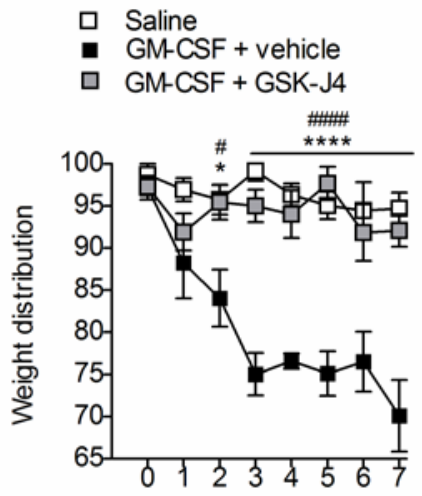

Time after i.a. injection (d)
C $\mathrm{mBSA} / \mathrm{TNF}$ arthritis

$\square$ Saline

- TNF + vehicle

$\square$ TNF + GSK-J4

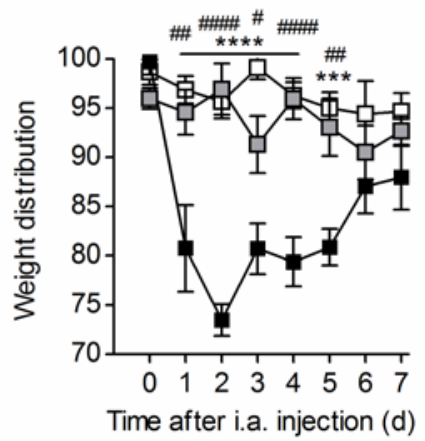

D mBSA/GM-CSF and mBSA/TNF arthritis
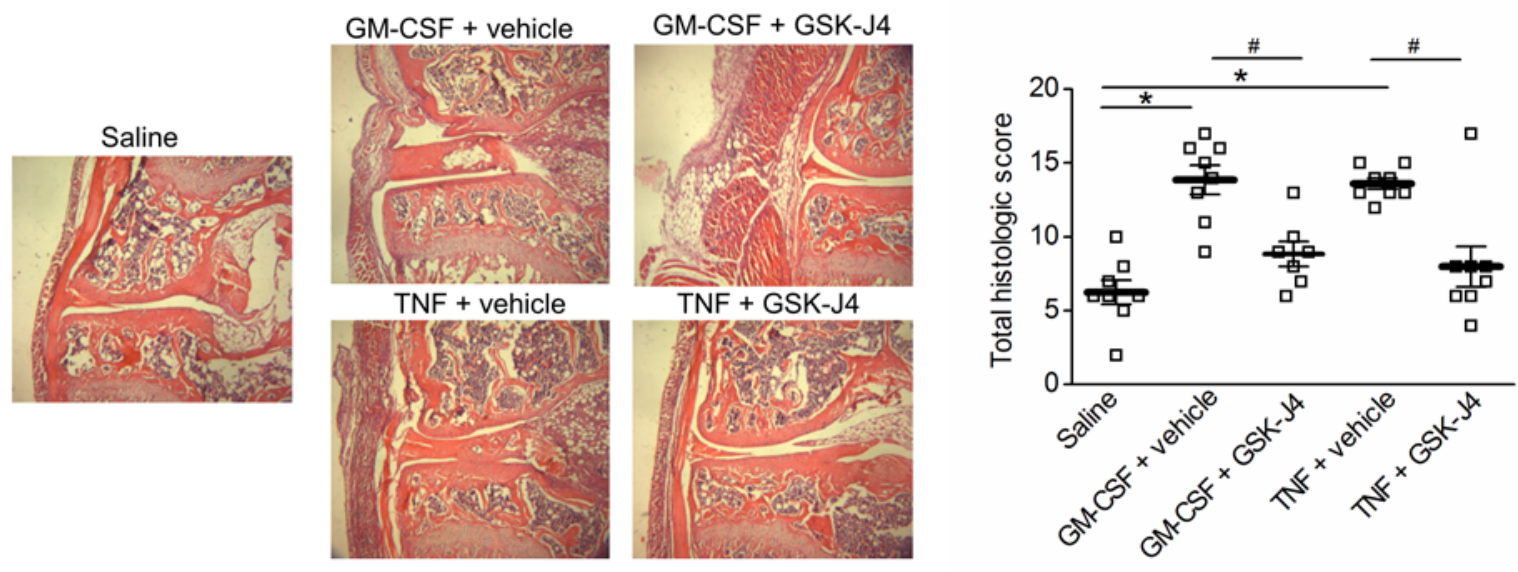

Figure 10. Inhibition of JMJD3 demethylase ameliorates TNF-driven inflammatory pain, and GM-CSF- and TNF-driven arthritic pain and disease. (A) Intraplantar (i.pl.) injection of TNF (20 ng) or saline in WT mice treated with vehicle or GSK-J4 (25 mg/kg i.p. at $t=-30$ minutes). Pain was measured ( $n=4-7$ mice/group). (B-D) Methylated BSA (mBSA)/granulocyte macrophage-colony stimulating factor (GM-CSF) arthritis and mBSA/TNF arthritis were induced in WT mice treated with vehicle or GSK-J4 (0.5 mg/kg i.p. daily from day -2 to day 2). (B and C) Pain and (D) arthritis (histology) were measured ( $n=7-8$ mice/group). Original magnification, $x 60$. Results are shown as mean $\pm \mathrm{SEM}$. $P$ values were obtained using a 2 -way ANOVA test for pain and a 1-way (D) ANOVA test for histology quantification. ${ }^{*} P<0.05,{ }^{* *} P<0.001,{ }^{* * *} P<0.0001$, saline versus TNF or GM-CSF + vehicle; ${ }^{\#} P<0.05,{ }^{\#} P<0.01$, \#\#\#\# $P<0.0001$, vehicle versus GSK-J4.

require mechanistically the same pathway as for GM-CSF-driven pain and arthritis, involving CCL17 formation via JMJD3-regulated IRF4 production (23).

Zymosan is a well-studied inflammogen used often to generate peripheral inflammatory pain in rodents as well as joint hypernociception and arthritis (24,29-33). TLR2/MyD88 signaling, complement, neutrophils and eicosanoids, as well as a number of cytokines, have been implicated in these functions $(23,24,31-33)$. We reported recently that the GM-CSF $\rightarrow$ CCL17 pathway is critical for zymosan-induced inflammatory pain, as well as ZIA pain and disease (23) - our findings above indicate that TNF is also linked with this pathway in these models in both the paw and the joint. In ZIA, the mAb neutralization data indicate that TNF, GM-CSF, and CCL17 are all required early to initiate the responses; however, unlike TNF, GM-CSF and CCL17 are still needed for the maintenance of pain and disease in this lymphocyte-independent model (23). In line with these data, we have previously suggested that GM-CSF is required for the maintenance of inflammatory peritonitis (34). Consistent with our data, in ZIA and various arthritis models TNF is usually concluded to have early effects on arthritis onset, including in one study on pain induction where delayed anti-TNF $\mathrm{mAb}$ had no effect (21,35-37); also, interestingly, in RA patients residual pain is often observed after anti-TNF therapy (38).

TNF-induced hyperalgesia upon i.pl. injection has been reported to be dependent on neutrophil migration to the hind paw and acts via TNF receptor type 1 (TNF-R1), but not through TNFR-R2 $(22,28)$; 
A

i.pl. CCL17

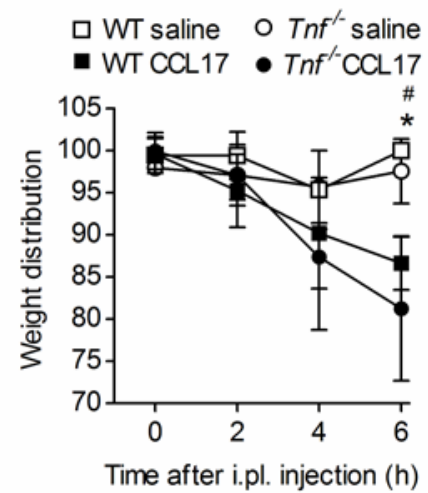

\section{B $\mathrm{mBSA} / \mathrm{CCL} 17$ arthritis}
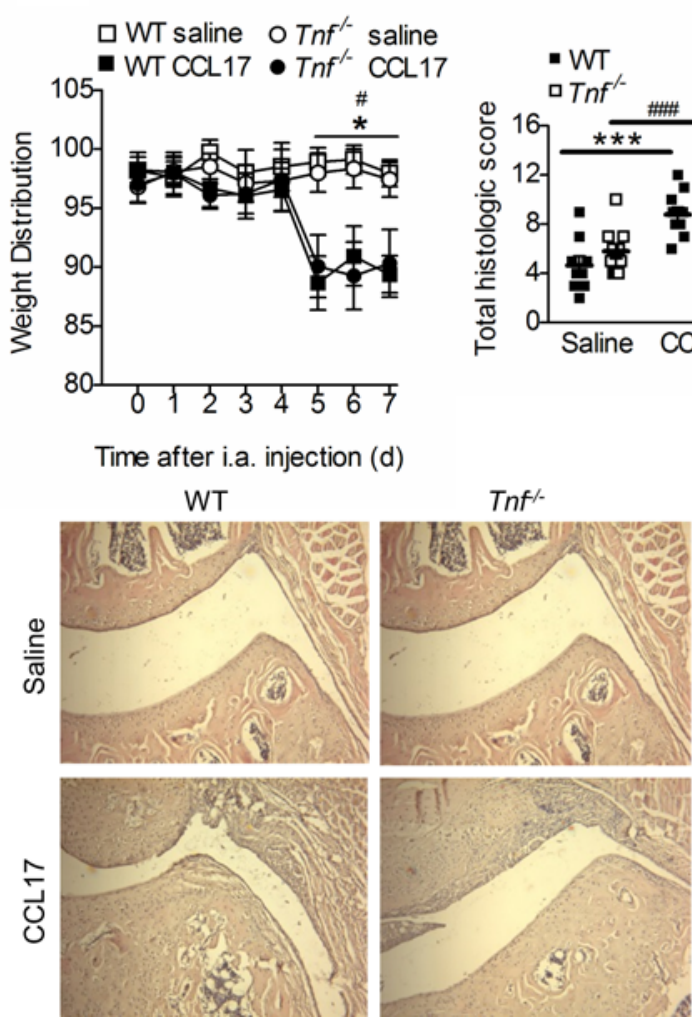

Figure 11. TNF is not required for CCL17-driven inflammatory pain and CCL17driven arthritic pain and disease. (A) Pain (incapacitance meter) was measured in WT and $\mathrm{Tnf}^{-/-}$mice following intraplantar (i.pl.) injection of CCL17 (50 ng) or saline ( $n=5$ mice/group). (B) Methylated BSA (mBSA)/CCL17 arthritis (intra-articular [i.a.] mBSA [day 0] and CCL17 or saline [days 0-2]) was induced in WT and Tnf ${ }^{-1-}$ mice and pain (incapacitance meter) and arthritis (histology) were measured ( $n=$ 6-10 mice/group). Original magnification, $x 125$. Results are shown as mean $\pm S E M$. $P$ values were obtained using 2 -way ANOVA test. ${ }^{*} P<0.05$, ${ }^{* * *} P<0.001$, WT

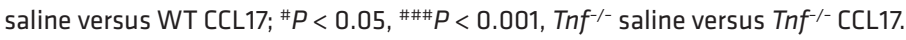

in addition to eicosanoids, a number of cytokines have been implicated $(21,22,39-42)$. We found above that such pain depends on GM-CSF and CCL17 and with the increased Ccl17 expression being GM-CSF dependent - in other words, GM-CSF and CCL17 form an essential part of the TNF-driven cytokine cascade and appear to be linked mechanistically via the activities of JMJD3 and IRF4. We thus propose from the data above that our GM-CSF $\rightarrow$ CCL17 pathway (23) also plays a key role in TNFinduced inflammatory pain.

Our mBSA/TNF arthritis model allowed us to demonstrate that systemically administered TNF can drive the GM-CSF $\rightarrow \mathrm{JMJD} 3 \rightarrow \mathrm{IRF} 4 \rightarrow \mathrm{CCL} 17$ pathway (23) in the joints, leading to pain and disease. It is noteworthy that the pain arising from exogenous TNF administration to the paw and to the mBSA-injected joint is dependent on the same pathway as well as on a contribution from a COX-2-dependent product(s). We have previously found that the mBSA/IL-1 arthritis model, which is driven by s.c. IL-1, is also GM-CSF dependent $(25,43)$ but, unlike the mBSA/TNF model, is $\mathrm{T}$ cell dependent (44), indicating that there are alternative pathways leading to a GM-CSF requirement. Interestingly, in the mBSA/TNF arthritis model, pain development, as measured by the incapacitance meter, was detected at 6 hours after s.c. TNF administration, which is faster than that developed in the mBSA arthritis models driven by s.c. IL-1, GM-CSF, and CCL17, in which it takes 2 to 5 days before pain can be detected using the same method $(23,25)$.

CCL17-driven inflammatory pain and mBSA/CCL17 arthritic pain and disease do not require TNF, as well as not requiring GM-CSF and IRF4 (23); however, a COX-2 dependence for the pain induction was previously indicated (23). Why downstream CCL17 should be so pivotal to the development of TNF- and GM-CSF-driven pain and disease is yet to be determined. TNF now can be added to the list of cytokines - for example, IL-4, IL-13, TSLP, and GM-CSF - able to enhance Ccl17 expression, albeit by an indirect mechanism involving GM-CSF in this

case (23). Traditionally, CCL17 has been considered a chemokine able to attract T cells $(45,46)$; however, the arthritis models studied above are T cell independent, suggesting other CCL17 functions (23, 47-49). CCL17 has been reported to be elevated in the sera or lesions of patients with many inflammatory disorders (50-59). Elevated CCL17 expression has been reported in dendritic cells from RA patients (60), and in Langerhans cells and keratinocytes in the skin of patients with acute and chronic atopic dermatitis (61). We again suggest that CCL17 can also be considered as a novel target for inflammatory pain management (23).

The cellular and molecular mechanisms governing inflammatory pain and disease are complex. TNF and GM-CSF can be key players in driving these pathologies $(1-4,38)$. Based on the reduction in the levels of proinflammatory cytokines, including GM-CSF, by neutralizing anti-TNF Abs in RA synovial cell cultures, it was proposed that a TNF-driven cytokine hierarchy exists, rather than positive feedback loops (7, $8)$, although this concept has been questioned $(36,62)$. Interestingly, in the context of our findings, high levels of circulating GM-CSF have recently been shown to correlate with responsiveness to anti-TNF agents in RA patients (9); also, in inflammatory bowel disease, a reduction in macrophage numbers and GMCSF gene expression correlated with a clinical response to anti-TNF mAb treatment (63). Despite GM-CSF 
being able to upregulate TNF expression in monocytes/macrophages, in particular at the transcript level $(11,23)$, and a reported synergistic relationship between GM-CSF and TNF in the in vitro development of dendritic cells (10), there have been no studies to our knowledge that have investigated any TNF dependency for the in vivo actions of GM-CSF. The in vivo data above suggest that a positive feedback loop involving GM-CSF and TNF can occur, which is in line with a cytokine network proposed previously $(2,16-18)$, thus making it difficult to determine whether GM-CSF is an upstream or downstream regulator of TNF in vivo.

It should be noted that we are not claiming that TNF is the only cytokine capable of linking up with GM-CSF biology and potentially with that of CCL17, nor that GM-CSF-mediated inflammation always involves TNF. IL-1, for example, can induce GM-CSF in a number of cell populations $(15,64)$, including T helper cells (65), and the T cell-dependent mBSA/IL-1 arthritis model is also GM-CSF dependent (43). $\mathrm{T}$ cells are often considered the important GM-CSF source in autoimmune and inflammation models (66, 67). Given that our mBSA/TNF model is T cell independent, a TNF-stimulated myeloid population(s) or a resident cell(s) (for example, endothelial cell and fibroblast) may be a source(s) of GM-CSF (2, 12, 13, 15-18). The cellular sources and targets of the cytokines forming the proposed nexus need to be defined, as does the link with COX-2 and its products. Type I interferon has been proposed to be linked importantly to TNF signaling (68). It would appear from our studies above that both GM-CSF and CCL17 can also be considered in this light.

In conclusion, we have shown here that in the models studied, TNF and GM-CSF act interdependently, but both are required; however, in the ZIA and mBSA/GM-CSF models TNF is not needed after initiation, unlike GM-CSF and CCL17. In spite of the differences between the biologies of GM-CSF and TNF, the development of TNF-driven inflammatory pain, as well as TNF-driven arthritic pain and disease, require the same downstream molecular pathway as GM-CSF-driven pain and arthritis, involving CCL17 formation via JMJD3-regulated IRF4 production (23). By no means are we implying that the nexus proposed is the only mechanism to account for the biologies of the respective cytokines. What we are proposing, however, is that if either TNF or GM-CSF is expressed at sufficient levels in an appropriate environment, then the nexus described might contribute significantly to the progression of an inflammatory reaction in that tissue. We have suggested previously that CCL17 may be a therapeutic target in inflammatory conditions where GM-CSF is important - we now suggest that this concept can be extended to include conditions in which the clinically important cytokine, TNF, is also involved.

\section{Methods}

Mice. GMCSF ${ }^{-1}$ mice, originally provided by the Ludwig Institute for Cancer Research, were as previously described $(23,25,69,70)$. C Cl1 $7^{E / E}$ have been described previously (45). Irf $4^{-/-}$mice were from Tak W. Mak (The Campbell Family Institute for Breast Cancer Research, University of Toronto, Toronto, Canada) (71), and Tnf'(72) and Rag1 ${ }^{-1-}$ (73) mice from The Walter and Eliza Hall Institute (WEHI). Mice were backcrossed onto the C57BL/ 6 background (WEHI) for more than 10 generations. Mice of both sexes (8-12 weeks old) were used.

Inflammatory pain models. Inflammatory pain was induced by i.pl. injection (10 $\mu 1)$ of either murine TNF (20 ng, R\&D Systems), murine GM-CSF (20 ng, R\&D Systems), murine CCL17 (50 ng, R\&D Systems), zymosan (100 $\mu \mathrm{g}$, Sigma-Aldrich), Complete Freund's adjuvant (CFA, Difco), or saline into the left hind footpad. Paw swelling was measured using spring callipers (Mitutoyo). Indomethacin (12.5 $\mu \mathrm{g} / \mathrm{paw}$, i.pl., Sigma-Aldrich) was given 2 hours after the inciting stimulus injection. The COX-2 inhibitor, SC58125 ( $5 \mathrm{mg} / \mathrm{kg}$, i.p., Tocris), and the JMJD3 inhibitor, GSK-J4 (25 mg/kg, i.p., Santa Cruz Biotechnology) were given 30 minutes prior to the inciting stimulus injection. Anti-TNF mAb (MP6-XT22, Schering BioPhar$\mathrm{ma})$, anti-GM-CSF mAb (22E9.11, Schering BioPharma) and isotype control mAbs were given i.pl. $(2 \mu \mathrm{g})$ at the same time as the indicated stimulus.

mBSA-induced arthritis models. Monoarticular arthritis was induced as before $(23,25)$ by i.a. injection of $100 \mu \mathrm{g}$ mBSA in $10 \mu \mathrm{l}$ saline into the right knee on day 0 , the left knee being injected with saline, followed by an s.c. injection, in the scruff of the neck on days 0-2, of either murine TNF (500 ng, R\&D Systems), murine GM-CSF (500 ng, R\&D Systems), murine CCL17 (600 ng, R\&D Systems), or saline. Mice were sacrificed (day 7) and knee joints collected for histology. Indomethacin (1 mg/kg, i.p.) was given once pain was evident. The JMJD3 inhibitor, GSK-J4 $(0.5 \mathrm{mg} / \mathrm{kg}$ i.p.), was given daily from day -2 to day 2 . AntiTNF mAb (MP6-XT22), anti-GM-CSF mAb (22E9.11), anti-CCL17 mAb (MAB529, clone 110904, R\&D Systems) and isotype control $\mathrm{mAbs}(150 \mu \mathrm{g}$ i.p.) were given on days -2 and 0 (prophylactic treatment) or once pain had developed (therapeutic treatment) (see Results section for treatment times). 
$Z I A$ model. For the induction of the ZIA model $(23,74)$, mice were injected with $300 \mu \mathrm{g}$ of sonicated zymosan (Sigma-Aldrich) in $10 \mu$ into the left knee joint, while the contralateral knee received saline as a control. On day 7, arthritic joints were collected for gene expression analysis using $\mathrm{PPCR}$ and histological analysis. Anti-TNF mAb (MP6-XT22), anti-CCL17 mAb (MAB529, clone 110904), and isotype control mAbs (150 $\mu$ g i.p.) were given prophylactically (days -2 and 0$)$ and/or therapeutically (day 1 ).

Pain readings. As an indicator of pain, the differential distribution of weight over a 3-second period between the inflamed paw or limb relative to the non-inflamed paw or limb was measured using the incapacitance meter (IITC Life Science Inc). This technique has previously been validated for measurement of both paw and arthritic knee pain $(23,25,27,69)$. Mice were acclimatized to the incapacitance meter on at least 3 occasions prior to the commencement of the experiment. Three measurements were taken for each time point and averaged.

Histology. At termination, the knee joints were removed, fixed, decalcified, and paraffin embedded $(23,25,69)$. Frontal sections $(7 \mu \mathrm{m})$ were stained with H\&E. For the mBSA/TNF, mBSA/GM-CSF, and mBSA/CCL17 models, cellular infiltration, synovitis (synovial hyperplasia), pannus formation, cartilage damage, and bone erosions were scored separately from 0 (normal) to 5 (severe) as described previously $(23,25)$. For ZIA, cell infiltration, proteoglycan loss (Safranin O/fast green stain), and bone erosions were scored separately from 0 (normal) to 3 (severe) as before $(23,74)$.

$q P C R$. qPCR experiments were performed as described previously (23). Briefly, total RNA was extracted using an Isolate II RNA Mini Kit (Bioline) and reverse transcribed using SuperScript III reverse transcriptase (Life Technologies). qPCR was carried out using an ABI PRISM 7900HT sequence detection system (Applied Biosystems) and predeveloped TaqMan probe/primer combinations for murine GMCSF, Ccl17, Illb, Tnf, and ubiquitin C (Ubc) (Life Technologies). All samples were assayed in duplicate. Threshold cycle numbers were transformed to $\Delta \mathrm{Ct}$ values, and the results were expressed relative to a reference gene, $U b c$.

Isolation and culture of murine monocyte/macrophage cell populations. Bone marrow monocytes were isolated by sorting $\mathrm{CD} 115^{+} \mathrm{CD} 11 \mathrm{~b}^{+}$bone marrow cells from WT C57BL/6 mice, using anti-CD115 (ASF98) and anti-CD11b (M1/70) (BD Biosciences) mAbs. Day 4 thioglycolate-elicited peritoneal macrophages from WT and $\mathrm{Tnf}^{\prime-}$ mice were prepared as before (75). Cells were treated with either murine GM-CSF (20 $\mathrm{ng} / \mathrm{ml}$ ) or PBS, for the indicated time periods, in the presence or absence of anti-TNF mAb (MP6-XT22, 1 $\mathrm{ng} / \mathrm{ml}$ ) (Schering BioPharma) (76) or IgG1 isotype control mAb $(1 \mathrm{ng} / \mathrm{ml})$.

ELISA. Secreted murine CCL17 was measured by ELISA as per the manufacturer's instructions (R\&D Systems).

Statistics. For mRNA expression, a 2-tailed Student's $t$ test or a 2-way ANOVA was used. For pain readings, a 1-way ANOVA or 2-way ANOVA was used, and for histologic scores, the Mann-Whitney 2-sample rank test, 1-way ANOVA, or 2-way ANOVA was used (Prism version 5.04, GraphPad Software). Bonferroni's post hoc test was used when appropriate. Data were plotted as mean \pm SEM with significance $P$ values as indicated. A $P$ value less than 0.05 was considered significant.

Study approval. All animal experiments were approved by The University of Melbourne Animal Ethics committee.

\section{Author contributions}

A.D. Cook and JAH conceived the study. A.D. Cook, MCL, RS, HK, A.D. Christensen, AA, AJF, and DCL conducted the investigation. JES and IF provided resources. A.D. Cook, MCL, and RS performed formal analysis. A.D. Cook and JAH wrote the original draft of the manuscript. A.D. Cook, JES, and IF reviewed and edited the manuscript. A.D. Cook, DCL, and JAH supervised the study.

\section{Acknowledgments}

A. Achuthan and J.A. Hamilton were supported in part by a grant from GSK. A.D. Cook and J.A. Hamilton were supported by a grant (1043147), and J.A. Hamilton by a Senior Principal Research Fellowship from the National Health and Medical Research Council of Australia. A.D. Christensen was supported by a Postdoc Fellowship from Novo Nordisk A/S, Denmark. I. Förster was supported by the Deutsche Forschungsgemeinschaft IRTG 2168 (DFG).

Address correspondence to: John A. Hamilton, University of Melbourne, Department of Medicine, Royal Melbourne Hospital, Parkville, Victoria 3050, Australia. Phone: 61.3.8344.5480; Email: jahami@unimelb.edu.au. 
1. Noack M, Miossec P. Selected cytokine pathways in rheumatoid arthritis. Semin Immunopathol. 2017;39(4):365-383

2. Hamilton JA. Colony-stimulating factors in inflammation and autoimmunity. Nat Rev Immunol. 2008;8(7):533-544.

3. Hamilton JA. GM-CSF as a target in inflammatory/autoimmune disease: current evidence and future therapeutic potential. Expert Rev Clin Immunol. 2015;11(4):457-465.

4. Hamilton JA, Cook AD, Tak PP. Anti-colony-stimulating factor therapies for inflammatory and autoimmune diseases. Nat Rev Drug Discov. 2016;16(1):53-70.

5. Burmester GR, et al. A randomised phase IIb study of mavrilimumab, a novel GM-CSF receptor alpha monoclonal antibody, in the treatment of rheumatoid arthritis. Ann Rheum Dis. 2017;76(6):1020-1030.

6. Cook AD, Louis C, Robinson MJ, Saleh R, Sleeman MA, Hamilton JA. Granulocyte macrophage colony-stimulating factor receptor $\alpha$ expression and its targeting in antigen-induced arthritis and inflammation. Arthritis Res Ther. 2016;18(1):287.

7. Haworth C, Brennan FM, Chantry D, Turner M, Maini RN, Feldmann M. Expression of granulocyte-macrophage colonystimulating factor in rheumatoid arthritis: regulation by tumor necrosis factor-alpha. Eur J Immunol. 1991;21(10):2575-2579.

8. Monaco C, Nanchahal J, Taylor P, Feldmann M. Anti-TNF therapy: past, present and future. Int Immunol. 2015;27(1):55-62.

9. Bystrom J, et al. Response to treatment with TNF $\alpha$ inhibitors in rheumatoid arthritis is associated with high levels of GM-CSF and GM-CSF ${ }^{+}$T lymphocytes. Clin Rev Allergy Immunol. 2017;53(2):265-276.

10. Lehner M, et al. Autocrine TNF is critical for the survival of human dendritic cells by regulating BAK, BCL-2, and FLIPL. J Immunol. 2012;188(10):4810-4818.

11. Hart PH, Whitty GA, Piccoli DS, Hamilton JA. Synergistic activation of human monocytes by granulocyte-macrophage colony-stimulating factor and IFN-gamma. Increased TNF-alpha but not IL-1 activity. J Immunol. 1988;141(5):1516-1521.

12. Munker R, Gasson J, Ogawa M, Koeffler HP. Recombinant human TNF induces production of granulocyte-monocyte colonystimulating factor. Nature. 1986;323(6083):79-82.

13. Broudy VC, Kaushansky K, Segal GM, Harlan JM, Adamson JW. Tumor necrosis factor type alpha stimulates human endothelial cells to produce granulocyte/macrophage colony-stimulating factor. Proc Natl Acad Sci USA. 1986;83(19):7467-7471.

14. Alsalameh S, Firestein GS, Oez S, Kurrle R, Kalden JR, Burmester GR. Regulation of granulocyte macrophage colony stimulating factor production by human articular chondrocytes. Induction by both tumor necrosis factor-alpha and interleukin 1, downregulation by transforming growth factor beta and upregulation by fibroblast growth factor. J Rheumatol. 1994;21(6):993-1002.

15. Leizer T, Cebon J, Layton JE, Hamilton JA. Cytokine regulation of colony-stimulating factor production in cultured human synovial fibroblasts: I. Induction of GM-CSF and G-CSF production by interleukin-1 and tumor necrosis factor. Blood. 1990;76(10):1989-1996.

16. Hamilton JA. A colony-stimulating factor network involving mononuclear phagocytes and other cells. In: van Furth R, ed. Haematopoietic Growth Factors and Mononuclear Phagocytes. Basel, Swizterland: Karger;1993:29-35.

17. Hamilton JA. Rheumatoid arthritis: opposing actions of haemopoietic growth factors and slow-acting anti-rheumatic drugs. Lancet. 1993;342(8870):536-539.

18. Hamilton JA. GM-CSF in inflammation and autoimmunity. Trends Immunol. 2002;23(8):403-408.

19. Wicks IP, Roberts AW. Targeting GM-CSF in inflammatory diseases. Nat Rev Rheumatol. 2016;12(1):37-48.

20. Stösser S, Schweizerhof M, Kuner R. Hematopoietic colony-stimulating factors: new players in tumor-nerve interactions. $J M o l$ Med. 2011;89(4):321-329.

21. Cunha FQ, Poole S, Lorenzetti BB, Ferreira SH. The pivotal role of tumour necrosis factor alpha in the development of inflammatory hyperalgesia. Br J Pharmacol. 1992;107(3):660-664.

22. Cunha TM, Verri WA, Silva JS, Poole S, Cunha FQ, Ferreira SH. A cascade of cytokines mediates mechanical inflammatory hypernociception in mice. Proc Natl Acad Sci USA. 2005;102(5):1755-1760.

23. Achuthan A, et al. Granulocyte macrophage colony-stimulating factor induces CCL17 production via IRF4 to mediate inflammation. J Clin Invest. 2016;126(9):3453-3466

24. Guerrero AT, et al. Toll-like receptor 2/MyD88 signaling mediates zymosan-induced joint hypernociception in mice: participation of TNF- $\alpha$, IL-1 $\beta$ and CXCL1/KC. Eur J Pharmacol. 2012;674(1):51-57.

25. Cook AD, et al. Granulocyte-macrophage colony-stimulating factor is a key mediator in inflammatory and arthritic pain. Ann Rheum Dis. 2013;72(2):265-270.

26. Lee MC, et al. G-CSF receptor blockade ameliorates arthritic pain and disease. J Immunol. 2017;198(9):3565-3575.

27. Inglis JJ, et al. Regulation of pain sensitivity in experimental osteoarthritis by the endogenous peripheral opioid system. Arthritis Rheum. 2008;58(10):3110-3119.

28. McNamee KE, et al. IL-17 induces hyperalgesia via TNF-dependent neutrophil infiltration. Pain. 2011;152(8):1838-1845.

29. Blom AB, van Lent PL, Holthuysen AE, van den Berg WB. Immune complexes, but not streptococcal cell walls or zymosan, cause chronic arthritis in mouse strains susceptible for collagen type II auto-immune arthritis. Cytokine. 1999;11(12):1046-1056.

30. Meller ST, Gebhart GF. Intraplantar zymosan as a reliable, quantifiable model of thermal and mechanical hyperalgesia in the rat. Eur J Pain. 1997;1(1):43-52.

31. Turnbach ME, Randich A. The effect of spinal and systemic administration of indomethacin on zymosan-induced edema, mechanical hyperalgesia, and thermal hyperalgesia. J Pain. 2001;2(1):25-35.

32. Guerrero AT, et al. Involvement of LTB4 in zymosan-induced joint nociception in mice: participation of neutrophils and PGE2. J Leukoc Biol. 2008;83(1):122-130.

33. Kanashiro A, Figueiredo MJ, Malvar Ddo C, Souza GE. Cytokines, but not corticotropin-releasing factor and endothelin-1, participate centrally in the febrile response in zymosan-induced arthritis in rats. Brain Res. 2015;1610:12-19.

34. Lenzo JC, et al. Control of macrophage lineage populations by CSF-1 receptor and GM-CSF in homeostasis and inflammation. Immunol Cell Biol. 2012;90(4):429-440.

35. Ferreira SH, Lorenzetti BB, Poole S. Bradykinin initiates cytokine-mediated inflammatory hyperalgesia. Br J Pharmacol. 1993;110(3):1227-1231.

36. Joosten LA, Helsen MM, van de Loo FA, van den Berg WB. Anticytokine treatment of established type II collagen-induced arthritis in DBA/1 mice. A comparative study using anti-TNF alpha, anti-IL-1 alpha/beta, and IL-1Ra. Arthritis Rheum. 
1996;39(5):797-809

37. Inglis JJ, Nissim A, Lees DM, Hunt SP, Chernajovsky Y, Kidd BL. The differential contribution of tumour necrosis factor to thermal and mechanical hyperalgesia during chronic inflammation. Arthritis Res Ther. 2005;7(4):R807-R816.

38. Udalova I, Monaco C, Nanchahal J, Feldmann M. Anti-TNF therapy. Microbiol Spectr. 2016;4(4).

39. Woolf CJ, Allchorne A, Safieh-Garabedian B, Poole S. Cytokines, nerve growth factor and inflammatory hyperalgesia: the contribution of tumour necrosis factor alpha. Br J Pharmacol. 1997;121(3):417-424.

40. Cunha TM, et al. Crucial role of neutrophils in the development of mechanical inflammatory hypernociception. $J$ Leukoc Biol. 2008;83(4):824-832.

41. Segond von Banchet G, Boettger MK, König C, Iwakura Y, Bräuer R, Schaible HG. Neuronal IL-17 receptor upregulates TRPV4 but not TRPV1 receptors in DRG neurons and mediates mechanical but not thermal hyperalgesia. Mol Cell Neurosci. 2013;52:152-160.

42. Verri WA, Cunha TM, Parada CA, Poole S, Cunha FQ, Ferreira SH. Hypernociceptive role of cytokines and chemokines: tar gets for analgesic drug development? Pharmacol Ther. 2006;112(1):116-138.

43. Yang YH, Hamilton JA. Dependence of interleukin-1-induced arthritis on granulocyte-macrophage colony-stimulating factor Arthritis Rheum. 2001;44(1):111-119.

44. Lawlor KE, Campbell IK, O'Donnell K, Wu L, Wicks IP. Molecular and cellular mediators of interleukin-1-dependent acute inflammatory arthritis. Arthritis Rheum. 2001;44(2):442-450.

45. Alferink J, et al. Compartmentalized production of CCL17 in vivo: strong inducibility in peripheral dendritic cells contrasts selective absence from the spleen. J Exp Med. 2003;197(5):585-599.

46. Iellem A, et al. Unique chemotactic response profile and specific expression of chemokine receptors CCR 4 and CCR 8 by CD4(+)CD25(+) regulatory T cells. J Exp Med. 2001;194(6):847-853.

47. Weber C, et al. CCL17-expressing dendritic cells drive atherosclerosis by restraining regulatory T cell homeostasis in mice. $J$ Clin Invest. 2011;121(7):2898-2910.

48. Heiseke AF, et al. CCL17 promotes intestinal inflammation in mice and counteracts regulatory T cell-mediated protection from colitis. Gastroenterology. 2012;142(2):335-345.

49. Stutte S, et al. Requirement of CCL17 for CCR7- and CXCR4-dependent migration of cutaneous dendritic cells. Proc Natl Acad Sci USA. 2010;107(19):8736-8741.

50. Jugde F, et al. Quantitation of chemokines (MDC, TARC) expression in mucosa from Crohn's disease and ulcerative colitis. Eur Cytokine Netw. 2001;12(3):468-477.

51. Ye Y, et al. Serum chemokine CCL17/thymus activation and regulated chemokine is correlated with coronary artery diseases. Atherosclerosis. 2015;238(2):365-369.

52. Okamoto H, Koizumi K, Yamanaka H, Saito T, Kamatani N. A role for TARC/CCL17, a CC chemokine, in systemic lupus erythematosus. J Rheumatol. 2003;30(11):2369-2373.

53. Wang J, et al. Circulating levels of Th1 and Th2 chemokines in patients with ankylosing spondylitis. Cytokine. 2016;81:10-14.

54. Hampel U, Sesselmann S, Iserovich P, Sel S, Paulsen F, Sack R. Chemokine and cytokine levels in osteoarthritis and rheumatoid arthritis synovial fluid. J Immunol Methods. 2013;396(1-2):134-139.

55. Hillen MR, Moret FM, Lafeber FPJG, Hack CE, Radstake TRDJ, van Roon JAG. Targeting CD1c-expressing Mdcs to inhibit Tcell activation and thymus and activation regulated chemokine (TARC)-dependent chemotaxis in ra. Arthritis Rheum. 2013;65(Suppl 10):S406.

56. Narikawa K, Misu T, Fujihara K, Nakashima I, Sato S, Itoyama Y. CSF chemokine levels in relapsing neuromyelitis optica and multiple sclerosis. J Neuroimmunol. 2004;149(1-2):182-186.

57. Kataoka Y. Thymus and activation-regulated chemokine as a clinical biomarker in atopic dermatitis. J Dermatol. 2014;41(3):221229

58. Staples KJ, Hinks TS, Ward JA, Gunn V, Smith C, Djukanović R. Phenotypic characterization of lung macrophages in asthmatic patients: overexpression of CCL17. J Allergy Clin Immunol. 2012;130(6):1404-12.e7.

59. García JJ, Cidoncha A, Bote ME, Hinchado MD, Ortega E. Altered profile of chemokines in fibromyalgia patients. Ann Clin Biochem. 2014;51(Pt 5):576-581.

60. Radstake TR, et al. Increased expression of CCL18, CCL19, and CCL17 by dendritic cells from patients with rheumatoid arthritis, and regulation by Fc gamma receptors. Ann Rheum Dis. 2005;64(3):359-367.

61. Kakinuma T, et al. Thymus and activation-regulated chemokine in atopic dermatitis: Serum thymus and activation-regulated chemokine level is closely related with disease activity. J Allergy Clin Immunol. 2001;107(3):535-541.

62. Campbell IK, Roberts LJ, Wicks IP. Molecular targets in immune-mediated diseases: the case of tumour necrosis factor and rheumatoid arthritis. Immunol Cell Biol. 2003;81(5):354-366.

63. Caprioli F, et al. Reduction of $\mathrm{CD} 8^{+}$macrophages and decreased IL-17 expression in intestinal mucosa of patients with inflam matory bowel disease strongly correlate with endoscopic response and mucosal healing following infliximab therapy. Inflamm Bowel Dis. 2013;19(4):729-739.

64. Campbell IK, Novak U, Cebon J, Layton JE, Hamilton JA. Human articular cartilage and chondrocytes produce hemopoietic colony-stimulating factors in culture in response to IL-1. J Immunol. 1991;147(4):1238-1246.

65. Lawlor KE, Wong PK, Campbell IK, van Rooijen N, Wicks IP. Acute CD4 ${ }^{+}$T lymphocyte-dependent interleukin-1-driven arthritis selectively requires interleukin-2 and interleukin-4, joint macrophages, granulocyte-macrophage colony-stimulating factor, interleukin-6, and leukemia inhibitory factor. Arthritis Rheum. 2005;52(12):3749-3754.

66. Becher B, Tugues S, Greter M. GM-CSF: from growth factor to central mediator of tissue inflammation. Immunity. 2016;45(5):963-973

67. Campbell IK, et al. Differentiation of inflammatory dendritic cells is mediated by NF-kB1-dependent GM-CSF production in CD4 T cells. J Immunol. 2011;186(9):5468-5477.

68. Yarilina A, Ivashkiv LB. Type I interferon: a new player in TNF signaling. Curr Dir Autoimmun. 2010;11:94-104

69. Cook AD, et al. Granulocyte-macrophage colony-stimulating factor is a key mediator in experimental osteoarthritis pain and disease development. Arthritis Res Ther. 2012;14(5):R199. 
70. Louis C, et al. Specific contributions of CSF-1 and GM-CSF to the dynamics of the mononuclear phagocyte system. J Immunol. 2015;195(1):134-144.

71. Mittrücker HW, et al. Requirement for the transcription factor LSIRF/IRF4 for mature B and T lymphocyte function. Science. 1997;275(5299):540-543.

72. Körner H, et al. Distinct roles for lymphotoxin-alpha and tumor necrosis factor in organogenesis and spatial organization of lymphoid tissue. Eur J Immunol. 1997;27(10):2600-2609.

73. Mombaerts P, Iacomini J, Johnson RS, Herrup K, Tonegawa S, Papaioannou VE. RAG-1-deficient mice have no mature B and T lymphocytes. Cell. 1992;68(5):869-877.

74. Keystone EC, Schorlemmer HU, Pope C, Allison AC. Zymosan-induced arthritis: a model of chronic proliferative arthritis following activation of the alternative pathway of complement. Arthritis Rheum. 1977;20(7):1396-1401.

75. Cook AD, Braine EL, Hamilton JA. The phenotype of inflammatory macrophages is stimulus dependent: implications for the nature of the inflammatory response. J Immunol. 2003;171(9):4816-4823.

76. Abrams JS, Roncarolo MG, Yssel H, Andersson U, Gleich GJ, Silver JE. Strategies of anti-cytokine monoclonal antibody development: immunoassay of IL-10 and IL-5 in clinical samples. Immunol Rev. 1992;127:5-24. 